\title{
VANISHING THEOREM FOR AN ELLIPTIC DIFFERENTIAL OPERATOR
}

\author{
BOHUMIL CENKL
}

\section{INTRODUCTION}

In the paper [12] D.C. Spencer defines the resolution

$$
0 \rightarrow \Theta \rightarrow \underline{\mathrm{C}^{0}} \stackrel{D}{\rightarrow} \underline{\mathrm{C}^{1}} \stackrel{D}{\rightarrow} \cdots \stackrel{D}{\rightarrow} \underline{\mathrm{C}^{\mathrm{n}}} \rightarrow 0
$$

of the sheaf $\Theta$ of germs of solutions of a regular elliptic system of linear partial differential equations on a differentiable manifold $M$ by jet forms $C^{r}$, which are in a canonical way associated with the given system. This resolution is a generalization of the classical de Rham resolution, and of the Dolbeault resolution of the sheaf of germs of holomorphic functions on a complex manifold $M$. The laplacian $D D^{*}+D^{*} D$, where $D^{*}$ is the adjoint of $D$ with respect to some chosen metric, is elliptic if and only if the original system of partial differential equations is elliptic.

$$
\text { Let } \begin{gathered}
C_{0}=\oplus_{r} C^{2 r}, C_{1}=\oplus_{r} C^{2 r+1} . \text { Then } \\
D+D^{*}: C_{0} \rightarrow C_{1}
\end{gathered}
$$

and the adjoint operator, which also is denoted $D+D^{*}$, maps $C_{1}$ into $C_{0}$. If $M$ is compact (closed), the system is elliptic and the resolution exact, the analytic index of the operator $D+D^{*}$ is therefore the Euler-Poincare characteristic $\chi(M, \Theta)$, where

$$
\chi(M, \Theta)=\sum_{r=0}^{n}(-1)^{r} \operatorname{dim} \boldsymbol{H}^{r}(\boldsymbol{M}, \Theta),
$$

and $n$ is the dimension of the manifold $M$. By the Atiyah-Singer theorem the topological index $i_{t}\left(D+D^{*}\right)$ of the differential operator $D+D^{*}$, where $D+D^{*}$ is an elliptic operator, is equal to the Euler-Poincare characteristic. In his paper [7], Kodaira gives sufficient conditions which a complex line bundle over a complex compact manifold $M$ should satisfy to force the vanishing in positive degrees of the cohomology groups with values in the sheaf

Communicated by D. C. Spencer, July 13, 1967. This work was partly supported by the National Science Foundation under Grant GP-4172. 
of germs of holomorphic sections. The method of the proof is similar to that used by S. Bochner, Curvature and Betti numbers, Ann. of Math. 49 (1948) 379-390, and Curvature and Betti numbers, II, Ann. of Math. 50 (1949) 77-93.

In this paper, sufficient conditions are given for the vanishing of the cohomology groups $H^{r}(M, \Theta), r>0$, where $M$ is a compact differentiable manifold and $\Theta$ is the sheaf of germs of solutions of a homogeneous regular elliptic system $\mathscr{D}$ on $M$ mapping the sections of one vector bundle into the sections of another. The condition is stated in terms of sufficient positivity of the curvature of the operator $\mathscr{D}$, which is a generalization of the conditon given by Kodaira in terms of the characteristic class of a complex line bundle over a complex manifold $M$. Using the expression for the Euler-Poincare characteristic we obtain from the vanishing theorem the relation between the dimension of the space of global solutions of the regular elliptic system on $M$ and topological invariants of $M$ and $\mathscr{D}$.

To $\mathscr{D}$ there is canonically associated a vector bundle $R$ over $M$. If we introduce a riemannian metric along the fibres of the vector bundle $R$ and on $M$, we obtain another resolution, the so-called " $\beta$-resolution"

$$
0 \rightarrow \Theta \rightarrow \underline{\mathrm{B}^{0}} \stackrel{\beta D_{0}}{\longrightarrow} \underline{\mathrm{B}}^{1} \stackrel{\beta D_{0}}{\longrightarrow} \underline{\mathrm{B}^{2}} \stackrel{\beta D_{0}}{\longrightarrow} \cdots \stackrel{\beta D_{0}}{\longrightarrow} \underline{\mathrm{B}}^{\mathrm{n}} \rightarrow 0
$$

of the sheaf $\Theta$, which is in some sense simpler than the one above. The sections of $B^{r}$ are differential forms with coefficients in a vector bundle, and $\beta D_{0}$ is a first order differential operator. This resolution is equivalent to the original one so far as exactness is concerned, and if the differential equation is the Cauchy-Riemann equation on a complex manifold $M$, the $\beta$-resolution reduces to the classical Dolbeault resolution. Theorem 3.3 gives sufficient conditions for the vanishing of $H^{r}(M, \Theta), r>0$, in terms of the $\beta$-sequence, and then the dimension of $H^{0}(M, \Theta)$ is equal to the topological index $i_{t}\left(\beta D_{0}+D_{0}^{*}\right)$.

The author wishes to express his gratitude to D.C. Spencer for many helpful discussions, comments and suggestions during preparation of this paper.

\section{NOTATION AND FUNDAMENTAL NOTIONS}

\section{Jet bundles}

Let $M$ be a $C^{\infty}$-manifold of dimension $n$, and let $E$ and $F$ be vector bundles over $M$ with fibre dimensions $m$ and $l$. For each $x \in M$ let us identify smooth (i.e. $C^{\infty}$ ) sections of $E$ at $x$ if their Taylor expansions agree up to the order $\mu$. We call an equivalence class a jet of order $\mu$ at the point $x$ and denote by $J_{\mu}(E)_{x}$ the vector space of these equivalence classes. Moreover, $J_{\mu}(E)$ $=\bigcup_{x \in M} J_{\mu}(E)_{x}$ is called the bundle of $\mu$-jets of $E$. If we denote by $E$ the sheaf 
of sections of $E$, we have the natural map

$$
j_{\mu}: \underline{\mathrm{E}} \rightarrow \underline{\mathrm{J}_{\mu}(\mathrm{E})}
$$

which, to a section $s$ of $E$ and a point $x \in M$, associates the $\mu$-jet of $s$ at $x$.

If $S^{\mu}\left(T^{*}\right)$ is the $\mu$-th symmetric product of the cotangent bundle $T^{*}$ of $M$, we see that the following sequence is exact, for all $\mu \geq 0$,

$$
0 \rightarrow E \otimes S^{\mu+1}\left(T^{*}\right) \stackrel{\gamma}{\rightarrow} J_{\mu+1}(E) \stackrel{\pi}{\rightarrow} J_{\mu}(E) \rightarrow 0,
$$

where $\gamma$ is an injection and $\pi$ the ordinary projection of jets.

Definition 2.1. The jet differential is a first-order differential operator

$$
D: J_{\mu+1}(E) \otimes A^{r} T^{*} \rightarrow J_{\mu}(E) \otimes \Lambda^{r+1} T^{*}
$$

having the following properties:

$$
D(\sigma \wedge \eta)=D \sigma \wedge \eta+(-1)^{r} \pi \sigma \wedge d \eta
$$

for $\sigma \in \underline{\mathrm{J}_{\mu+1}(\mathrm{E}) \otimes \Lambda^{r} \mathrm{~T}^{*}}$, and any real-valued 1-form $\eta$ on $\mathrm{M}$,

(ii) the sequence

$$
\underline{\mathrm{E}} \stackrel{j_{\mu+1}}{\longrightarrow} \underline{\mathrm{J}_{\mu+1}(\mathrm{E})} \stackrel{D}{\longrightarrow} \underline{\mathrm{J}_{\mu}(\mathrm{E}) \otimes \mathrm{T}^{*}}
$$

is exact for all non-negative integers $r, \mu$.

The operator $D$ is uniquely determined by (i) and (ii) and $D^{2}=0$ (see [13], [14]). In terms of a local coodinate $\left(x^{1}, \cdots, x^{n}\right)$ covering the neighborhood $U$ on $M$, we consider a local section $\sigma$ of $J_{\mu+1}(E) \otimes \Lambda^{r} T^{*}$ over $U$; then $\sigma=\left\{\sigma_{q}|| q \mid \leq \mu+1\right\}$ where $q=\left(q_{1}, \cdots, q_{k}, \cdots, q_{n}\right)$ is an ordered $n$-tuple of non-negative integers $q_{k},|q|=q_{1}+\cdots+q_{n}$, and $\sigma_{q}=\left\{\sigma_{q}^{j} \mid 1 \leq j \leq m\right\}$ where

$$
\sigma_{q}^{j}=\frac{1}{r !} \sum \sigma_{q, i_{1} \cdots i_{r}}^{j} d x^{i_{1}} \wedge \cdots \wedge d x^{i_{r}}
$$

The jet differential operator $D$ applied to $\sigma$ has the form

$$
D \sigma=d \pi \sigma-\delta \sigma,
$$

where $d$ is the ordinary differential, and $\delta$ the so-called formal differential. The formal differential $\delta$ is a mapping given by

$$
(\delta \sigma)_{q}=\sum_{j=1}^{n} d x^{j} \wedge \sigma_{q+1_{j}},
$$


BOHUMIL CENKL

where $q+1_{j}=\left(q_{1}, \cdots, q_{j-1}, q_{j}+1, q_{j+1}, \cdots, q_{n}\right)$. Let us notice that $\delta^{2}=\delta \delta=0$, and that $\delta$ is a bundle map (linear over the functions).

The operator $\delta$ is actually defined by $D$ in the sense that the following diagram is commutative :

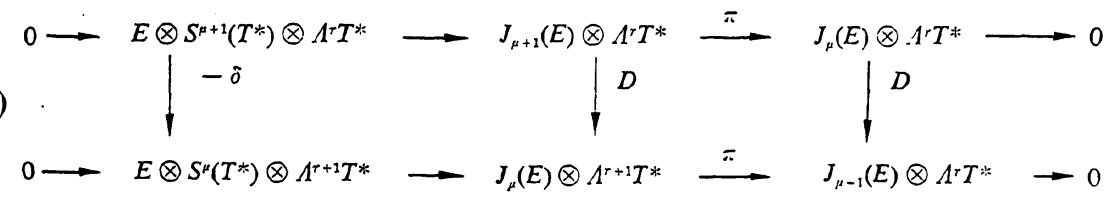

From (2.1) and the definition of the operator $\delta$ we see that the sequence $E \otimes S^{\mu+1}\left(T^{*}\right) \otimes \Lambda^{r-1} T^{*} \stackrel{\delta}{\longrightarrow} E \otimes S^{\mu}\left(T^{*}\right) \otimes \Lambda^{r} T^{*} \stackrel{\delta}{\longrightarrow} E \otimes S^{\mu-1}\left(T^{*}\right) \otimes \Lambda^{r-1} T^{*}$ is exact. Let us consider a sequence $\mathscr{A}=\left\{\mathscr{A}^{\mu, r}\right\}$ of sub-bundles $\mathscr{A}^{\mu, r} \subset E \otimes S^{\mu}\left(T^{*}\right) \otimes \Lambda^{r} T^{*}$ such that $\delta \mathscr{A}^{\mu, r} \subset \mathscr{A}^{\mu-1, r+1}$. The sequence $\mathscr{A}^{\mu+1, r-1} \stackrel{\delta}{\longrightarrow} \mathscr{A}^{\mu, r} \stackrel{\delta}{\longrightarrow} \mathscr{A}^{\mu-1, r+1}$ is not exact in general, but we still have $\delta^{2}=0$. The corresponding cohomology will be called the $\delta$-cohomology of the sequence $\left\{\mathscr{A}^{\mu, r}\right\}$ and the cohomology groups are denoted by $H^{\mu, r}(\mathscr{A})$.

\section{Differential equations}

A differential operator of order $\mu_{0}$ from $E$ to $F$ is a sheaf map $\mathscr{D}: \underline{\mathrm{E}} \rightarrow \underline{\mathrm{F}}$ which is locally defined as follows. Let $U$ be a coordinate neighborhood and let $E|U \cong U \times V, F| U \cong U \times W$ be trivializations of the corresponding vector bundles. Then, for a section $s$ of $E \mid U$,

$$
\mathscr{D}_{s}=\sum_{\mid \alpha: \leq \mu_{0}} a_{\alpha} D^{\alpha} s, \alpha=\left(\alpha_{1}, \cdots, \alpha_{n}\right)
$$

where the $a_{\alpha}$ are smooth $(l \times m)$-matrix-valued functions, and

$$
D^{\alpha}=\frac{\partial^{\alpha}}{\left(\partial x^{1}\right)^{\alpha_{1}} \cdots\left(\partial x^{n}\right)^{\alpha_{n}}}
$$

in terms of the coordinates in $U$.

The mapping $\mathscr{D}$ can be factorized through $J_{\mu_{0}}(E)$ in the sense that there exists a unique bundle map $\rho_{\mu_{0}}(\mathscr{D})=\rho_{\mu_{0}}: J_{\mu_{0}}(E) \rightarrow F$ such that the following diagram commutes :

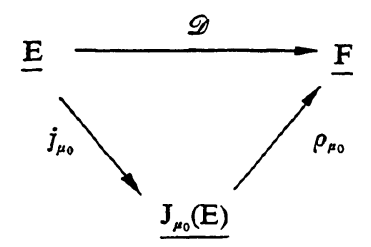


The differential operator $\mathscr{D}_{\nu}: \mathrm{E} \rightarrow \mathrm{J}_{\nu}(\mathrm{F})$ given by $\mathscr{D}_{\nu}=j_{\nu} \circ \mathscr{D}$ is of order $\nu+\mu_{0}$, and is the so-called $\nu$-th prolongation of $\mathscr{D}$.

Definition 2.2. A system of partial differential equations of order $\mu_{0}$ given by $\mathscr{D}$ on $M$ is the kernel $R_{\mu_{0}}$ of the map $\rho_{\mu_{0}}$ in the exact sequence

$$
0 \longrightarrow R_{\mu_{0}} \longrightarrow J_{\mu_{0}}(E) \stackrel{\rho_{\mu_{0}}}{\longrightarrow} F \text {. }
$$

A solution of the system $R_{\mu_{0}}$ is a smooth section $f$ of $E$ such that $j_{\mu_{0}}(f)$ is a section of $R_{\mu_{0}}$ over $M$. A system of partial differential equations given by the prolonged operator $\mathscr{D}_{\nu}$ is of order $\mu_{0}+\nu$ and is the kernel $R_{\mu_{0}+\nu}$ of the map $\rho_{\mu_{0}+\nu}$ following in the diagram:

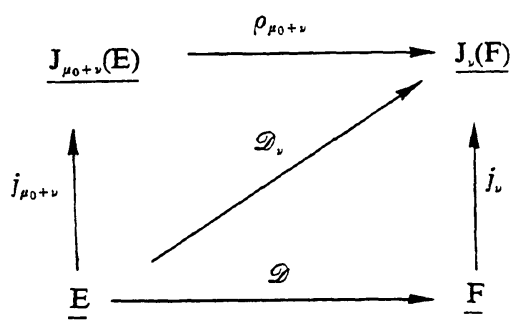

Definition 2.3. The differential operator $\mathscr{D}$ (and also the corresponding system of partial differential equations) is said to be regular if

(i) for each $\mu \geq \mu_{0}$, the $R_{\mu}$ are vector bundles over $M$;

(ii) for each $\mu \geq \mu_{0}$, the map $\pi: R_{\mu+1} \rightarrow R_{\mu}$ is subjective.

The bundle $R_{\mu}$ can be "trivialized" by choice of linearly independent sections of $R_{\mu}$ over a suitable neighborhood $U$ on $M$. Whenever we speak about trivialization of $R_{\mu}$, we have in mind this procedure.

The condition (ii) in Definition 2.3 actually requires that the homogeneous equation $\mathscr{D} S=0$ has formal solutions [10].

Let $\xi \in T_{X}^{*}, x \in M$, be a non-zero cotangent vector. We define the symbol $\sigma(\mathscr{D}, \xi)$ of $\mathscr{D}$ at $\xi$ to be the composed map

$$
E_{x} \rightarrow E_{x} \otimes S^{\mu_{0}}\left(T_{x}^{*}\right) \stackrel{r}{\rightarrow} J_{\mu_{0}}(E)_{x} \stackrel{\rho_{\mu_{0}}}{\rightarrow} F_{x}
$$

The first map is given by $e \rightarrow \frac{1}{\mu_{0} !}\left(e \otimes \xi^{\mu_{0}}\right)$. Then, in terms of a local coordinate $\left(x^{1}, \cdots, x^{n}\right)$ on $U$, with $\xi=\xi_{1} d x^{1}+\cdots+\xi_{n} d x^{n}$, the symbol $\sigma(\mathscr{D}, \xi): E_{x} \rightarrow F_{x}$ is given by the formula

$$
\sigma(\mathscr{D}, \xi) e=\sum_{|\alpha|=\mu_{0}} a_{\alpha}(x) \xi^{\alpha} e .
$$

Now let us consider the exact sequence of vector bundles for $\mu \geq \mu_{0}$ 


$$
0 \rightarrow g_{\mu}^{r} \rightarrow R_{\mu}^{r} \stackrel{\pi}{\rightarrow} J_{\mu-1}(E) \otimes \Lambda^{r} T^{*} \rightarrow 0,
$$

where $R_{\mu}^{r}=R_{\mu} \otimes \Lambda^{r} T^{*}$ and $g_{\mu}^{r}=g_{\mu} \otimes \Lambda^{r} T^{*}$. The subbundle $g_{\mu}^{r}$ of $R_{\mu}^{r}$ is defined by this sequence. It is obvious that $g_{\mu}^{r}$ is a subbundle of $E \otimes S^{\mu}\left(T^{*}\right) \otimes$ $\Lambda^{r} T^{*}$. In terms of the sequence $g=\left\{g_{\mu}^{r}\right\}, \mu \geq \mu_{0}, r \geq 0$, we have the following definition for the system of equations to be involutive (see [13]).

Definition 2.4. The system of regular partial differential equations $R_{\mu}$ (or the operator $\mathscr{D}_{\mu}$ ) is said to be involutive if the cohomology groups $H^{\nu, r}(g)$ vanish for $\nu \geq \mu+1$ and all $r$.

Remark (see [3], [11]). (i) The vanishing of $H^{\nu, r}(g)$ for $\nu \geq \mu+1, r=$ $0,1,2, \ldots$ is equivalent to the exactness of the sequence

$$
0 \rightarrow g_{\nu+n} \stackrel{\delta}{\rightarrow} g_{\nu+n-1}^{1} \stackrel{\delta}{\rightarrow} \cdots \stackrel{\delta}{\rightarrow} g_{\nu}^{n} \rightarrow 0, \quad \nu \geq \mu .
$$

(ii) If $\mathscr{D}$ is a regular differential operator of order $\mu_{0}$, then there exists $\mu_{1}$ depending on $n=\operatorname{dim} M, m=\operatorname{dim} E$ and $\mu_{0}$ such that $H^{\mu, r}(g)=0$ for $\mu \geq \mu_{1}$ and all $r$ ( $\delta$-Poincaré lemma).

\section{Spencer's resolution}

Let us denote by $\Theta$ the sheaf of germs of solutions of the homogeneous equation $\mathscr{D} f=0$. We shall describe the construction, due essentially to $\mathbf{K}$. Bott [2], of Spencer's resolution

$$
0 \rightarrow \Theta \stackrel{\iota}{\rightarrow} \underline{\mathrm{C}_{\mu}^{0}} \stackrel{D}{\rightarrow} \underline{\mathrm{C}_{\mu}^{1}} \stackrel{D}{\rightarrow} \underline{\mathrm{C}_{\mu}^{2}} \stackrel{D}{\rightarrow} \ldots \stackrel{D}{\rightarrow} \underline{\mathrm{C}_{\mu}^{\mathrm{n}}} \rightarrow 0
$$

of the sheaf $\Theta$ by sheaves $\underline{C}_{\mu}^{r}$ of jet forms of order $\mu \geq \mu_{1}$ and degrees $r=$ $0,1, \cdots, n$.

For $\mu \geq \mu_{1}$, let

$$
A_{\mu}^{r}=\left\{\xi \in g_{\mu}^{r} \mid \delta \zeta=0\right\}
$$

for all non-negative integers $r$. Then $A_{\mu}^{r}$ is a subbundle of $R_{\mu}^{r}$. Let us define the vector bundles

$$
C_{\mu}^{r}=R_{\mu+1}^{r} / A_{\mu+1}^{r} .
$$

We see that, for $\mu \geq \mu_{1}$, we have the first-order differential operator

$$
D^{\prime}: R_{u+1}^{r} \rightarrow C_{\mu}^{r+1}
$$

given by the commutative diagram 


$$
\begin{aligned}
& 0 \rightarrow \frac{\mathrm{g}_{\mu+2}^{\mathrm{r}}}{\downarrow-\delta} \rightarrow \frac{\mathrm{R}_{\mu+2}^{\mathrm{r}}}{\downarrow D} \rightarrow \frac{\mathrm{R}_{\mu+1}^{\mathrm{r}}}{\downarrow D^{\prime}} \rightarrow 0 \\
& 0 \rightarrow \underline{\mathrm{A}_{\mu+1}^{\mathrm{r}+1}} \rightarrow \underline{\mathrm{R}_{\mu+1}^{\mathrm{r}+1}} \stackrel{p}{\rightarrow} \underline{\mathbf{R}_{\mu}^{\mathrm{r}+1}} \rightarrow 0 .
\end{aligned}
$$

The mapping $D^{\prime}$ factors through $C_{\mu}^{r}$ in the sense that there is a unique map $\mathrm{D}: \mathrm{C}_{\mu}^{\mathrm{r}} \rightarrow \mathrm{C}_{\mu}^{\mathrm{r}+1}$ such that $D^{\prime}=D p$. We have then the resolution (2.7), where $C_{\mu}^{0}=R_{\mu+1}$ and 6 is the inclusion map. The resolution (2.7) is not always exact; actually the question of exactness is discussed in part III of this paper, and it is one of the main problems. The operator $D$ in $(2.7)$ is different from the one defined by (2.1). Whenever we use the operator (2.1) in what follows it will be stated explicitly, to avoid misunderstanding. It is useful to have an explicit description of $C_{\mu}^{r}$.

Proposition 2.1. Each element $u \in C_{\mu}^{r}, \mu \geq \mu_{1}, r \geq 0$ can be represented as a pair $(\sigma, \xi)$ such that $\sigma=\pi \rho, \xi=\delta \rho$ for some element $\rho \in R_{\mu+1}^{r}$, and $D u=(d \sigma-\xi,-d \xi)$. Moreover, $D^{2}=0$.

Proof. If we take any element $\rho \in R_{\mu+1}^{r}$ which represents a class in $R_{\mu+1}^{r} / A_{\mu+1}^{r}$, we get the same pair $(\pi \rho, \delta \rho)$, because any other element of the same equivalence class has the form $\rho+\zeta, \zeta \in A_{\mu+1}^{r} \subset g_{\mu+1}^{r}$ and then $\pi \rho=$ $\pi(\rho+\zeta), \delta \rho=\delta(\rho+\zeta)$.

From the diagram (2.8) we see that, if $\sigma \in R_{\mu+1}^{r}$, then $D^{\prime} \sigma=(d \pi \sigma-\delta \sigma$, $-d \delta \sigma$ ) because $d \delta=-\delta d$, where we consider the representation of the elements of $C_{\mu}^{r}$ by pairs. Let

$$
p: \sigma \rightarrow(\pi \sigma, \delta \sigma) .
$$

Since $D$ is defined by $D^{\prime}=D p$, we have $D u=D(\sigma, \xi)=(d \sigma-\xi,-d \xi)$. It follows at once that $D^{2}=0$.

This representation of the elements of $C_{\mu}^{r}$ is not very useful if the adjoint of the operator $D$ (with respect to some metric) is to be considered. This will be discussed later. We shall identify $u \in C_{\mu}^{r}$ with a pair of independent elements.

Proposition 2.2. To a given splitting $\lambda: R_{\mu}^{r} \rightarrow R_{\mu+1}^{r}$ of the exact sequence

$$
0 \rightarrow g_{\mu+1}^{r} \rightarrow R_{\mu+1}^{r} \rightarrow R_{\mu}^{r} \rightarrow 0
$$

there corresponds an isomorphism $C_{\mu}^{r} \cong R_{\mu}^{r} \oplus A_{\mu}^{r+1}$, and

$$
D u=\left(D_{0} \sigma-\zeta, D_{0}\left(D_{0} \sigma-\zeta\right)\right),
$$

where $u=(\sigma, \zeta) \in R_{\mu}^{r} \oplus A_{\mu}^{r+1}, D_{0}=d-\delta \lambda$.

Proof. Let $u=(\sigma, \xi)$ be an element of $C_{\mu}^{r}$ which is represented as in Proposition 2.1. We see easily that the mapping $C_{\mu}^{r} \rightarrow R_{\mu}^{r} \oplus A_{\mu}^{r+1}$ defined by $(\sigma, \xi) \rightarrow(\sigma, \xi-\delta \lambda \sigma)$ is an isomorphism. Let $\zeta=\xi-\delta \lambda \sigma$. Then $D u=$ $D(\sigma, \xi)=D(\sigma, \zeta)=(d \sigma-\delta \lambda \sigma-\zeta,-d \xi-\delta \lambda(d \sigma-\delta \lambda \sigma-\zeta))=\left(D_{0} \sigma-\zeta\right.$, $\left.D_{0}\left(D_{0} \sigma-\zeta\right)\right)=D(\sigma, \zeta)$. 
Remark. The first-order differential operator $D_{0}=d-\delta \lambda$ defined on sections of $R_{\mu}^{r}$ is actually the covariant differential of a connection on the vector bundle $R_{\mu}^{r}$. In fact, for any element $\sigma \in \underline{\mathrm{R}_{\mu}^{\mathrm{r}}}$ and any function $f$ on $M$, we have $D_{0} f \sigma=f D_{0} \sigma+d f \otimes \sigma$. In terms of a trivialization of $R_{\mu}$ by choice of independent local sections, we can write $D_{0}=d+e(\Lambda)$, where $\Lambda$ is the local 1 -form of the connection and $e$ denotes the exterior multiplication.

Now let us introduce a riemannian metric along the fibres of the bundle $R_{\mu}$, where $\mu$ is a fixed integer, $\mu \geq \mu_{1}$. Then at each point $x \in M$ we have a scalar product $\langle\sigma, \tau\rangle_{x}$ of vectors $\sigma, \tau$ belonging to the fibre of $R_{\mu}$ over $x$. We also introduce a riemannian metric on $M$ (i.e. along the fibres of the tangent bundle $T$ of $M$ ), and denote by $\langle,\rangle_{x}$ the inner product on $R_{\mu}^{r}$ for $r=0,1$, $2, \cdots, n$; and by || $\mid x$ the corresponding norm. Denote by $B_{\mu}^{r}$ the orthogonal complement of the subbundle $A_{\mu}^{r}$ in $R_{\mu}^{r}$ with respect to this inner product. Then $B_{\mu}^{r}$ is a well-defined subbundle of $R_{\mu}^{r}$. Let us denote by $\alpha$ and $\beta$ the orthogonal projections of $R_{r}^{\mu}$ onto $A_{\mu}^{r}$ and $B_{\mu}^{r}$ respectively. We identify $C_{\mu}^{r}$ with $R_{\mu}^{r} \oplus A_{\mu}^{r+1}$, and write $\Lambda_{\mu}^{r}=A_{\mu}^{r} \oplus A_{\mu}^{r+1}$; we then have the exact sequence of vector bundles

$$
0 \rightarrow \Lambda_{\mu}^{r} \rightarrow C_{\mu}^{r} \rightarrow B_{\mu}^{r} \rightarrow 0 .
$$

Using the inner product along the fibres of $R_{\mu}^{r}$, and the splitting $\lambda$ of the exact sequence (2.9), we introduce the sequence

$$
0 \rightarrow \Theta \rightarrow \underline{\mathrm{B}_{\mu}^{0}} \stackrel{\beta D_{0}}{\longrightarrow} \underline{\mathrm{B}}_{\mu}^{1} \stackrel{\beta D_{0}}{\longrightarrow} \cdots \stackrel{\beta D_{0}}{\longrightarrow} \underline{\mathrm{B}}_{\mu}^{\mathrm{n}} \rightarrow 0 .
$$

Theorem 2.1. The diagram

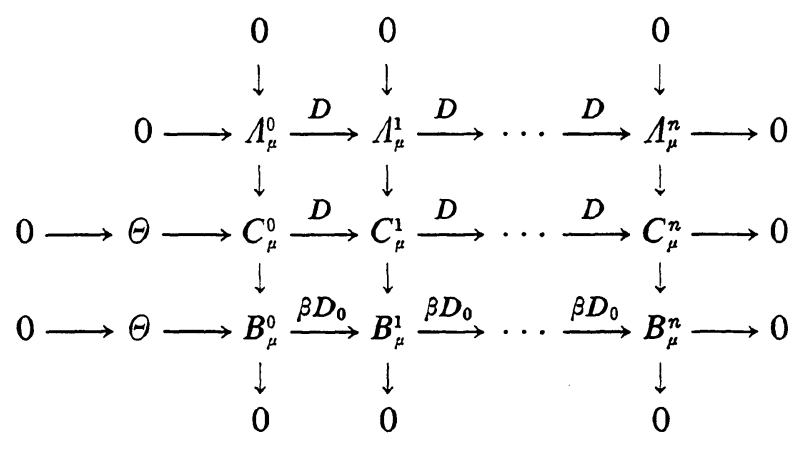

is commutative, and the last row is exact if and only if the middle one is. The first row is always exact.

Proof. Let $(\rho, \zeta) \in \Lambda_{\mu}^{r}$, and suppose that $D(\rho, \zeta)=0$, i.e., $\left(D_{0} \rho-\zeta\right.$, $\left.D_{0}\left(D_{0} \rho-\zeta\right)\right)=0$; then $(\rho, \zeta)=\left(\rho, D_{0}, 0\right)$. This implies that $-D(0, \rho)=(\rho, \zeta)$. Thus the first row of the diagram is exact at $\Lambda_{\mu}^{r}$ for $r \geq 1$. If $(\rho, \zeta) \in \underline{\Lambda_{\mu}^{0}}$, the 
assertion $D(\rho, \zeta)=0$ implies that $D_{0} \rho=\zeta$, where $\rho \in A_{\mu}^{0}$, i.e., $(\rho, \zeta)=(0,0)$, and the first row is an exact resolution of 0 . Since $D^{2}(\beta \sigma, 0)=0$, we see that $\left(\beta D_{0}\right)^{2}$ vanishes on sections of $B_{\mu}^{r}$.

From the assumption of exactness of the second or last row follow the exactness and commutativity of the diagram, since the vertical rows are exact by definition.

\section{Ellipticity}

In this section we shall discuss the concept of ellipticity and a fundamental inequality for an elliptic differential operator.

Let $\mathscr{D}: \underline{\mathrm{E}} \rightarrow \underline{\mathrm{F}}$ be a regular differential operator of order $\mu_{0}$, and let $R_{\mu}, g_{\mu}, \ldots$ be the corresponding vector bundles introduced above.

Definition 2.5. A non-zero cotangent vector $\xi \in T_{x}^{*}, x \in M$, is said to be noncharacteristic for $R_{\mu_{0}}$ (or $\left.\mathscr{D}\right)$ if the symbol map $\sigma(\mathscr{D}, \xi): E_{x} \rightarrow F_{x}$ is injective.

Remark. It is easy to see that the property of being noncharacteristic is independent of the prolongation of $\mathscr{D}[10]$.

Definition 2.6. The differential operator $\mathscr{D}$ is said to be elliptic at the point $x \in M$ if all the nonzero cotangent vectors $\xi \in T_{x}^{*}$ are noncharacteristic, and $\mathscr{D}$ is called an elliptic operator if it is elliptic at each point $x \in M$.

It is easily seen that locally the notion of ellipticity is the classical one. Let us consider the coordinate neighborhood $U$ and the trivialization $E|U, F| U$. We can then write the operator $\mathscr{D}: \mathrm{E}|\mathrm{U} \rightarrow \mathrm{F}| \mathrm{U}$ in the form (2.3). For $\xi=\xi_{1} d x^{1}+\cdots+\xi_{n} d x^{n}$ the symbol $\sigma(\mathscr{D}, \xi)$ has the form (2.6). The definition and (2.6) give the local meaning of ellipticity.

Proposition 2.3. The ellipticity of an operator $\mathscr{D}$ is equivalent to any one of the following properties:

(i) the symbol of $\omega$ in the following diagram is injective,

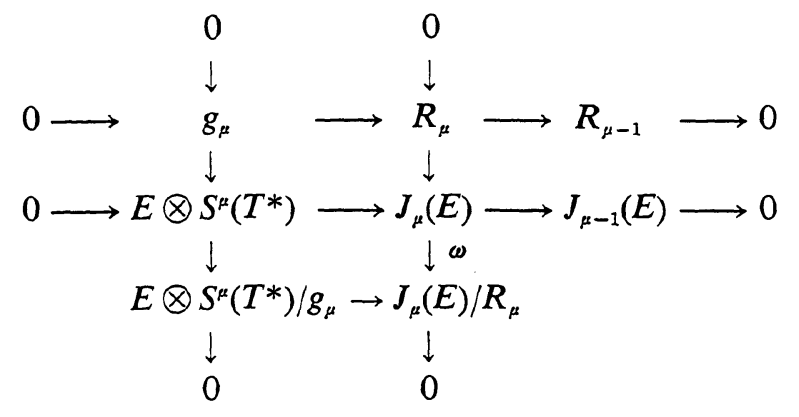

i.e., for every $\xi \in T_{x}^{*}, \xi \neq 0, x \in M$, the composition 


$$
E_{x} \stackrel{i(\xi)}{\longrightarrow} E_{x} \otimes S^{\mu}\left(T_{x}^{*}\right) \stackrel{r}{\longrightarrow} J_{\mu}(E) \stackrel{\omega}{\longrightarrow} J_{\mu}(E) / R_{\mu}
$$

is injective, where $i(\xi): e \rightarrow e \otimes \xi^{\mu}$;

(ii) the composition

$$
g_{\mu} \rightarrow E \otimes S^{\mu}\left(T^{*}\right) \rightarrow E \otimes S^{\mu}\left(T^{*}\right) / i(\xi) E
$$

is injective for each $\xi$;

(iii) for each $\xi$ the composition

$$
g_{\mu} \stackrel{\delta}{\rightarrow} g_{\mu-1} \otimes T^{*} \stackrel{\xi}{\rightarrow} g_{\mu-1} \otimes \Lambda^{2} T^{*}
$$

is injective;

(iv) the sequence

$$
0 \rightarrow \delta\left(g_{\mu}\right) \stackrel{\xi}{\rightarrow} \delta\left(g_{\mu} \otimes T^{*}\right)
$$

is exact for each nonzero $\xi, \mu \geq \mu_{1}$.

Proof. (i) follows immediately from the difinition.

(i) $\leftrightarrow$ (ii). From (i) it follows that for each $\xi$, the fibres $\gamma \circ i(\xi) E_{x}$ and $R_{\mu}$ over $x$ have only the 0-vector in common, because the composition $\omega \circ \gamma \circ i(\xi)$ is an injection and $\omega\left(\left.R_{\mu}\right|_{x}\right)=0$. Thus $i(\xi) E_{x}$ and $\left.g_{\mu}\right|_{x}$ have only zero in common, i.e., the mapping in (ii) is an injection. In order to prove the converse, let us assume that for some $\xi \in T_{x}^{*}$ and some $x \in M$ the mapping in (i) is not an injection. This means that, for some $e \in E_{x},\left.\gamma \circ i(\xi) e \in R_{\mu}\right|_{x}$, but from the above diagram it follows that $\left.\gamma \circ i(\xi) e \notin R_{\mu-1}\right|_{x}$. We conclude that $\left.i(\xi) e \in g_{\mu}\right|_{x}$ for that particular $\xi$ and some $e \in E_{x}$. This contradicts the injectivity of the mapping in (ii).

(ii) $\leftrightarrow$ (iii). Let us notice first that, for each $\xi \in T_{x}^{*}$, the kernel of the composition of the maps

$$
E_{x} \otimes S^{\mu}\left(T_{x}^{*}\right) \stackrel{\delta}{\rightarrow} E_{x} \otimes S^{\mu-1}\left(T_{x}^{*}\right) \otimes T_{x}^{*} \stackrel{\xi}{\rightarrow} E_{x} \otimes S^{\mu-1}\left(T_{x}^{*}\right) \otimes \Lambda^{2} T_{x}^{*}
$$

is $i(\xi) E_{x}$, and thus has only zero in common with $\left.g_{\mu}\right|_{x}$. This proves the necessity of (iii). The reverse follows by an argument similar to that used in the proof that (ii) implies (i).

(iii) $\leftrightarrow$ (iv). This equivalence is an obvious consequence of the fact that $\delta\left(g_{\mu}\right)$ is a subbundle of $g_{\mu-1}^{1}$ and $\delta\left(g_{\mu}^{1}\right)$ is a subbundle of $g_{\mu-1}^{2}$.

If $\mu \geq \mu_{1}$ we say that $\mu$ is in the stable range, and then we have the identity $\delta\left(g_{\mu}^{r}\right)=A_{\mu-1}^{r+1}$. The ellipticity of the involutive operator can be formulated in the following way ( $\mathscr{D}$ is involutive if $\mu_{1}=\mu_{0}+1$ ).

Proposition 2.4. The prolongation $\mathscr{D}_{\mu}, \mu \geq \mu_{1}$ of the operator $\mathscr{D}$ of order $\mu_{0}$ is an involutive operator, and it is an elliptic operator if and only if the diagram, for each nonzero cotangent vector $\xi \in T^{*}$, 


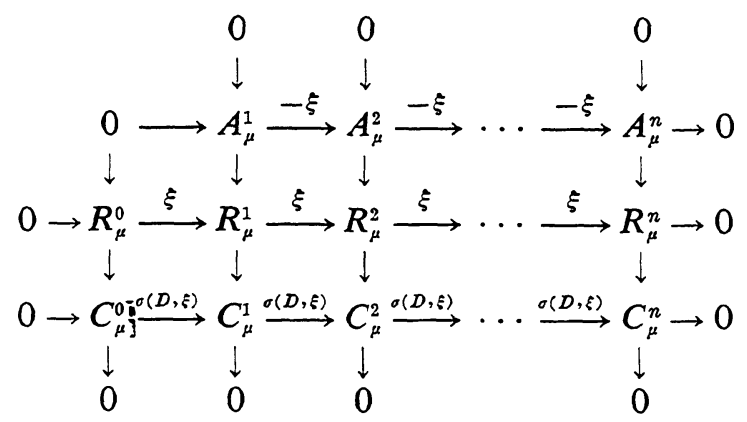

is exact and commutative for $\mu \geq \mu_{1}$.

Proof. It was proved by D. G. Quillen [10] that the last row of this diagram is exact if and only if the short sequence $0 \rightarrow C_{\mu}^{0} \stackrel{\sigma(D, \xi)}{\longrightarrow} C_{\mu}^{1}$ is exact. In terms of the representation $u=(\sigma, \zeta) \in R_{\mu}^{r} \oplus A_{\mu}^{r+1}$ of some element $u \in C_{\mu}^{r \mid}$, we can write explicitly $\sigma(D, \xi)(\sigma, \zeta)=(\xi \wedge \sigma,-\xi \wedge \zeta)$. Then the exactness of the short sequence $0 \rightarrow A_{\mu}^{1} \stackrel{-\xi}{\longrightarrow} A_{\mu}^{2}$ for all $\xi$ is equivalent to the exactness of the first row of the diagram. But the exactness of this short sequence for $\mu \geq \mu_{1}$ is equivalent to the ellipticity of the operator $\mathscr{D}$. Thus the ellipticity is equivalent to the exactness of the first row in the diagram. As was pointed out by $R$. Bott [2], the exactness of the first row is equivalent to the exactness of the bottom row, because the central sequence is always exact for each nonzero $\xi \in T^{*}$ and the vertical rows are exact by the definition.

Let $\tilde{\omega}: S^{*}=S^{*}(M) \rightarrow M$ be the unit cotangent sphere bundle. We then have the map $s: \tilde{\omega} A_{\mu}^{1} \rightarrow \tilde{\omega}^{*} A_{\mu}^{2}$ which, to an element $\lambda$ of the fibre lying over $\xi \in S^{*}$, associates an element $s(\lambda)=\xi \wedge \lambda$. In the stable range there is an injective map $\delta: \check{\omega}^{*} g_{\mu+1} \rightarrow \check{\omega}^{*} A_{\mu}^{1}$, and by the composition of these two maps we obtain the map $s \circ \delta: \tilde{\omega}^{*} g_{\mu+1} \rightarrow \tilde{\omega}^{*} A_{\mu}^{2}$.

Proposition 2.5. The operator $\mathscr{D}$ is elliptic if and only if the map $s \circ \delta$ defined above is injective.

Proof. This follows from Proposition 2.3 and the definition.

Now let us recall that the vector bundle $B_{\mu}^{r}$ is orthogonal to $A_{\mu}^{r}$ with respect to the inner product $\langle$,$\rangle on R_{\mu}^{r}$. Then the adjoint $\xi^{*}$ of the exterior left multiplication by any nonzero cotangent vector $\xi \in T_{x}^{*}$ of the elements of $\left.A_{\mu}^{r}\right|_{x}$ is well defined. Let us denote by $d^{*}$ the (formal) adjoint to $d$ with respect to the scalar product

$$
(,)=\int\langle,\rangle d M
$$

We can now state

Proposition 2.6 [12]. The ellipticity of an operator $\mathscr{D}$ is equivalent to the existence of a positive number $c$ such that, for each section $\zeta \in A_{\mu}^{r}, \mu>\mu_{1}$, 
$1 \leq r \leq n$, over any coordinate neighborhood $U \subset M$ with compact support, the following inequality holds:

$$
\left(\left\{d\left(\alpha d^{*}\right)+\left(\alpha d^{*}\right) d\right\} \zeta, \zeta\right) \geq c\|\zeta\|_{(1)}^{2}-\|\zeta\|^{2} .
$$

Remark. The norm \|\|$_{(s)}$ is the usual $s$-form in the Hilbert space $\mathscr{H}_{(s)}\left(M, C^{r}\right)$ defined, in the case of euclidean space, by the formula [6],

$$
\|u\|_{(s)}^{2}=(2 \pi)^{-n} \int\left(1+|\eta|^{2}\right)^{s}|\hat{u}(\eta)|^{2} d \eta,
$$

where $\hat{u}(\eta)$ is the Fourier transform of $u$. In the sequel we shall consider $\mathscr{H}_{0}\left(M, C^{r}\right)=L_{2}\left(M, C^{r}\right)$ as a Hilbert space with the product $()=$, $\int_{M}\langle\rangle d$,$M , and use the notation \|\|=\|\|_{(0)}$.

Proof (of Proposition 2.6). First let us remark that exactness of the first row of the diagram (2.13) is equivalent to the existence of a positive constant $c^{\prime}$ such that, for each nonzero $\xi \in T_{x}^{*}$ and any $\left.\lambda \in A_{\mu}^{r}\right|_{x}, 0 \leq r \leq n$, the inequality

$$
|\xi \lambda|_{x}^{2}+\left|\left(\alpha \xi^{*}\right) \lambda\right|_{x}^{2} \geq c^{\prime}|\xi|_{x}^{2}|\lambda|_{x}^{2}
$$

holds at each point $x \in M$. The norm ||$_{x}$ is defined by the inner product $\langle,\rangle_{x}$ in $\left.R_{\mu}\right|_{x}$, and $|\xi|_{x}^{2}=\xi_{1}^{2}(x)+\cdots+\xi_{n}^{2}(x)$, where $\xi=\xi_{1}(x) d x^{1}+\cdots+\xi_{n}(x) d x^{n}$. Using Fourier transforms, we obtain the quivalence of (2.14) and (2.15).

Proposition 2.7. The operator $\mathscr{D}$ is elliptic if and only if the sequence

$$
0 \rightarrow g_{\mu}^{0} \stackrel{\beta \xi}{\longrightarrow} \beta\left(g_{\mu}^{1}\right) \stackrel{\beta \xi}{\longrightarrow} \cdots \stackrel{\beta \xi}{\longrightarrow} \beta\left(g_{\mu}^{n}\right) \rightarrow 0
$$

is exact for each non-zero cotangent vector $\xi \in T^{*}$ and $\mu \geq \mu_{1}$.

Proof. The ellipticity of the operator $\mathscr{D}$ is equivalent to the exactness of the first row of the diagram

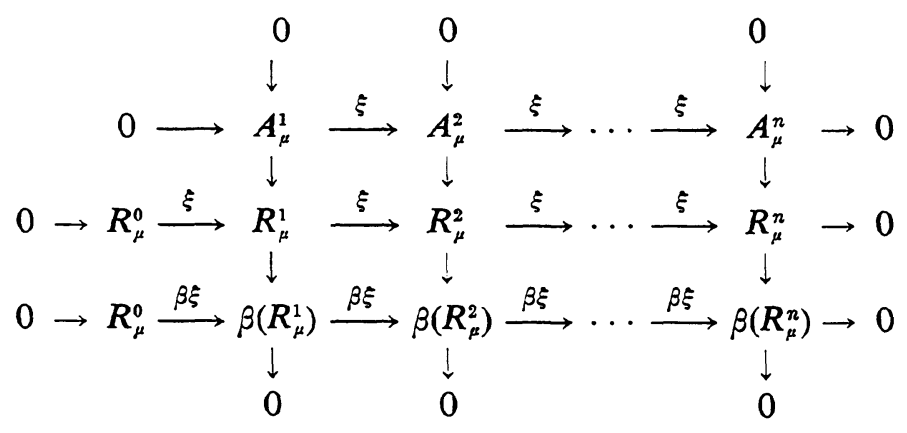

for each non-zero $\xi \in T_{x}^{*}$. The second row of this diagram is always exact; 
hence the last row is exact for all non-zero $\xi \in T_{x}^{*}$. Diagram chasing shows that ellipticity is equivalent to the exactness of the sequence $(2.16)$ as is easily seen from the following diagram:

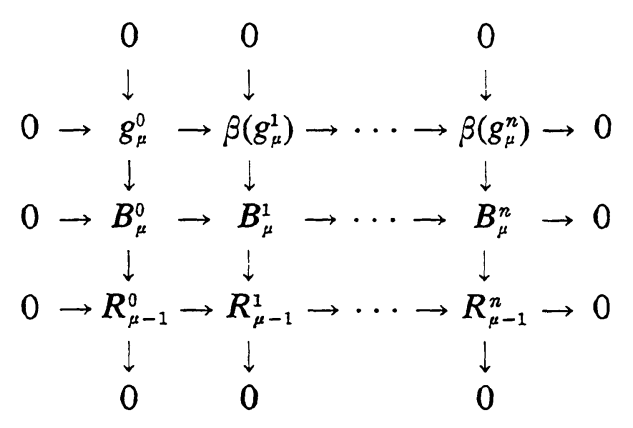

\section{VANISHING THEOREMS}

\section{Formalism of orthonormal frames}

This part is concerned with the differential geometric aspects of the bundles $R_{\mu}$. The forms and curvatures, which play an essential role in the vanishing theorems, are studied, together with the formulas for integration by parts in the orthonormal frame on the manifold $M$.

Let $M$ be a $C^{\infty}, n$-dimensional compact manifold. Let $\mathscr{D}$ be a regular elliptic differential operator of order $\mu_{0}$ from $E$ to $F$. Let $\mu$ be a fixed integer, $\mu \geq \mu_{1}$, and $R_{\mu}, g_{\mu}, \ldots$ the vector bundles, as defined in the previous part. We shall assume that there is introduced a riemannian metric into the fibres of $R_{\mu}$, and $\langle$,$\rangle denotes the corresponding inner product, and ($,$) the$ global scalar product on $M$.

Let us introduce a riemannian metric on the manifold $M$, and let $\left\{\omega^{1}, \cdots, \omega^{n}\right\}$ be the orthogonal coframe in the coordinate neighborhood $U \subset M$ with respect to the chosen metric. This means that

$$
\omega^{i} \cdot \omega^{k}=\delta_{k}^{i} ; \quad i, k=1,2, \cdots, n,
$$

where "." denotes the scalar product. We have

$$
\omega^{i}=a_{k}^{i} d x^{k}, \quad d x^{i}=b_{k}^{i} \omega^{k},
$$

where $a_{k}^{i}, b_{k}^{i}$ are $C^{\infty}$-functions such that $a_{j}^{k} b_{k}^{i}=\delta_{j}^{i}$. Let

$$
\frac{\partial}{\partial \omega^{i}}=b_{i}^{j} \frac{\partial}{\partial x^{j}}
$$

be the tangent vector, dual to $\omega^{i}$, i.e. 


$$
\omega^{i}\left(\frac{\partial}{\partial \omega^{j}}\right)=\delta_{j}^{i}
$$

But $\frac{\partial}{\partial \omega^{j}}$ also represents derivation in the exterior algebra of differential forms on $M$, in particular we have the formulas:

$$
\frac{\partial}{\partial \omega^{j}} \omega^{k}=c_{j i}^{k} \omega^{i}, c_{j i}^{k}=\frac{\partial a_{m}^{k}}{\partial x^{l}} b_{j}^{l} b_{i}^{m}
$$

To the given riemannian metric there is uniquely associated the metric connection, which can be given by the local 1 -form $\pi=\left(\pi_{k} \omega^{k}\right), \pi_{k}=\left(\pi_{j k}^{i}\right)$. Let us notice that $\pi_{j k}^{i}$ are symmetric in the lower indices if and only if $d \omega^{i}=0$; $i=1,2, \cdots, n$. This is seen from the relation between the connection symbols $\Gamma_{j k}^{i}, \Gamma_{j k}^{i}=\Gamma_{k j}^{i}$ in the coordinate system $\left(x^{1}, \cdots, x^{n}\right)$ and the symbols $\pi_{j k}^{i}$ in the orthonormal frame $\left\{\omega^{1}, \cdots, \omega^{n}\right\}$, namely

$$
\pi_{j k}^{i}=\Gamma_{m n}^{l} a_{l}^{i} b_{j}^{m} b_{k}^{n}-c_{j k}^{i} .
$$

Let us denote by $\nabla, \nabla=\omega^{i} \wedge \nabla_{i}$, the covariant differential with respect to this metric connection. If, for example, $\omega^{i}$ is considered as a section of the cotangent bundle $T^{*}(M)$, we have $\nabla \omega^{i}=d \omega^{i}+\pi_{j k}^{i} \omega^{j} \wedge \omega^{k}$. Since $\left\{\omega^{1}, \cdots, \omega^{n}\right\}$ is an orthonormal coframe, we get $\nabla \omega^{i}=0, i=1,2, \cdots, n$, or

$$
d \omega^{i}=\pi_{j k}^{i} \omega^{k} \wedge \omega^{j}
$$

The curvature from of the riemannian metric on $M$ is the 2-form $R=\nabla(\pi)$, $R=\left(R_{i j} \omega^{i} \wedge \omega^{j}\right), R_{i j}=\left(R_{l i j}^{k}\right)$.

We shall use the usual star operation "*" on forms with values in a vector bundle; * maps $r$-forms into $(n-r)$-forms. The symbol $\pi$ is used for interior product, for example, if $\varphi$ is a scalar $r$-form on $M$, then we denote by $\varphi \pi \frac{\partial}{\partial \omega^{k}}$ the $(r-1)$-form which is an interior product of $\varphi$ and $\frac{\partial}{\partial \omega^{k}}$. The volume element of $M$ will be denoted by

$$
*(1)=\omega^{1} \wedge \cdots \wedge \omega^{n} .
$$

Let $\left\{l_{1}(x), \cdots, l_{m}(x)\right\}$ be a frame in the fibre $R_{\mu}$ over $x \in U$. Then the choice of the frame $\left\{l_{1}(x), \cdots, l_{m}(x)\right\}$ in $\left.R_{\mu}^{r}\right|_{x}$ gives a trivialization of $R_{\mu}^{r}$ over

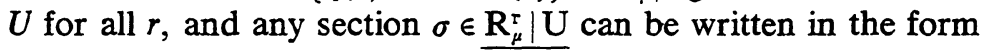

$$
\sigma=\frac{1}{r !} \sum_{i_{1}, \cdots, i_{r}} \sum_{\alpha} \sigma_{i_{1} \cdots i_{r}}^{\alpha} \omega^{i_{1}} \wedge \cdots \wedge \omega^{i_{r}} \otimes l_{\alpha}
$$

The matrix $a=a(x)=\left(a_{\alpha \beta}(x)\right)$ is the matrix of the metric tensor, with com- 
ponents $a_{\alpha \beta}(x)=\left\langle l_{\alpha}(x), l_{\beta}(x)\right\rangle$. Remember that the global product on $M$ is given by the formula

$$
(\sigma, \rho)=\int_{M}\langle\sigma, \rho\rangle *(1)=\int_{M} \sigma \wedge a * \rho
$$

for sections $\sigma, \rho$ of $R_{\mu}^{r}$. The 1 -form $\theta=\frac{1}{2} a^{-1} d a$, the connection form of the metric connection uniquely associated to the riemannian metric along the fibres of $R_{\mu}$. The curvature form of this connection is given by the formula

$$
\Pi=d \theta+\theta \wedge \theta \text {. }
$$

We have seen already that $\Lambda$ is the 1 -form of connection on $R_{\mu}$, which is given by the covariant differential $D_{0}=d+e(\Lambda)$. Let us define further the operators $D_{\Gamma}: \underline{\mathrm{R}_{\mu}^{\mathrm{r}}} \rightarrow \underline{\mathrm{R}_{\mu}^{\mathrm{r}+1}}$ by the formula

$$
D_{\Gamma}=D_{0}+e(\Gamma), \quad \Gamma=a^{-1} D_{0} a .
$$

The operator $D_{T}$ is again the covariant differential of some connection on $R_{\mu}$. Considering the commutator of $D_{0}$ and $D_{r}$, we define a local 2-form $\Omega$ by the formula

$$
\Omega \sigma=\left(D_{0} D_{\Gamma}+D_{\Gamma} D_{0}\right) \sigma,
$$

for each $\sigma \in \underline{\mathbf{R}_{\mu}^{\mathrm{r}}}$. Using the local orthonormal coframe introduced above, we have

$$
D_{\Gamma}=\omega^{j} \wedge \mathscr{D}_{j}, \quad D_{0}=\omega^{j} \wedge D_{j} .
$$

The formula (3.5) can be written explicitly in the form

$$
\left(\mathscr{D}_{j} D_{k}-D_{k} \mathscr{D}_{j}\right) \sigma_{i_{1} \cdots i_{r}}^{\alpha}=\Omega_{\beta j k}^{\alpha} \sigma_{i_{1} \cdots i_{r}}^{\beta},
$$

where $\sigma$ is given by (3.2).

Definition 3.1. The 2 -form $\Omega$ is called the curvature form of the operator $\mathscr{D}$ and the corresponding tensor the curvature tensor of $\mathscr{D}$.

Let $D_{0}^{*}$ be the formal adjoint operator to $D_{0}$ with respect to the global product (, ). Then for the "laplacian" $\square_{0}=D_{0} D_{0}^{*}+D_{0}^{*} D_{0}$ and any element $\sigma \in \mathbb{R}_{\mu}^{\mathrm{r}}$ the Weitzenbock formula can be written in the form

$$
\begin{aligned}
\left\{\left(D_{0} D_{0}^{*}+D_{0}^{*} D_{0}\right) \sigma\right\}_{i_{1} \cdots i_{r}}^{\alpha}= & -\sum_{j=1}^{n} \mathscr{D}_{j} D_{j} \sigma_{i_{1} \cdots i_{r}}^{\alpha} \\
& +\sum_{s=1}^{r}\left(\Omega_{\beta j i_{s}}^{\alpha}-\delta_{\beta}^{\alpha} R_{i_{s}}^{j}\right) \sigma_{i_{1} \cdots i_{s-1} j i_{s+1} \cdots i_{r}}^{\beta} \\
& -\frac{1}{2} \sum_{i_{\mu}, i_{\nu}} R_{i_{\mu} i_{\nu} j}^{l} \sigma_{i_{1} \cdots i_{\mu-1}}^{\alpha} l i_{\mu+1} \cdots i_{\nu-1} j i_{\nu+1} \cdots i_{r}
\end{aligned}
$$


where

$$
R_{i_{s}}^{j}=\sum_{k=1}^{n} R_{k i_{s} k}^{j}
$$

are components of the Ricci tensor. The formula (3.8) can be written symbolically in the form

$$
\square_{0} \sigma=-\sum \mathscr{D}_{j} D_{j} \sigma-\frac{1}{2} R_{\sigma}-\hat{R} \sigma+\Omega \sigma,
$$

where $R_{\sigma}, \hat{R}_{\sigma}, \Omega_{\sigma}$ are defined by (3.8) and (3.9).

Remark. If $D_{0}$ is the covariant differential of the metric connection associated to the riemannian metric along the fibres of $R_{\mu}$, i.e., if $\theta=\Lambda$, then $\Gamma=0, D_{\Gamma}=D_{0}$, and we get the usual Weitzenbock formula, which in the complex case is in [4]. But, in general, if the differential operator $\mathscr{D}$ and, therefore, $R_{\mu}$ is given, $A$ is not a 1 -form of any metric connection on $R_{\mu}$. Nevertheless, there are some special elliptic differential operators such that the splitting $\lambda$ of the sequence (2.9) can be chosen in such a way that $\Lambda$ is the 1 -form of the metric connection associated to the given riemannian metric along the fibres of $R_{\mu}$ (see III. 5, for example).

Let us now define the quadratic forms

$$
\begin{aligned}
& \Omega(\sigma, \sigma)=(\Omega \sigma, \sigma), \\
& R(\sigma, \sigma)=(R \sigma, \sigma), \\
& \hat{R}(\sigma, \sigma)=\left(\hat{R}_{\sigma}, \sigma\right) .
\end{aligned}
$$

Lemma 3.1. For each element $\sigma \in \underline{\mathrm{R}_{\mu}^{\mathrm{r}}}$ the following identity holds:

$$
\begin{aligned}
\sum_{j, k=1}^{n}\left(\left\{\mathscr{D}_{j} D_{k}-D_{k} \mathscr{D}_{j}\right\}\left(\sigma \pi \frac{\partial}{\partial \omega^{j}}\right), \sigma \pi \frac{\partial}{\partial \omega^{k}}\right) \\
=\Omega(\sigma, \sigma)-\hat{R}(\sigma, \sigma)-\frac{1}{2} R(\sigma, \sigma) .
\end{aligned}
$$

Proof. An element $\sigma \in \underline{\mathbf{R}_{\mu}^{\mathrm{r}}}$ can be written in the form (3.2); then

$$
\left(\sigma \pi \frac{\partial}{\partial \omega^{j}}\right)^{\alpha}=\frac{1}{(r-1) !} \sum \sigma_{j i_{1} \cdots i_{r-1}}^{\alpha} \omega^{i_{1}} \wedge \cdots \wedge \omega^{i_{r-1}}
$$

and if we denote by "; " the covariant derivative with respect to the connection given by the operator $D_{0}$, we have

$$
\begin{aligned}
\left\{\mathscr{D}_{j} D_{k}\left(\sigma \pi \frac{\partial}{\partial \omega^{j}}\right)\right\}^{\alpha}= & \frac{1}{(r-1) !} \sum\left\{\sigma_{j i_{1} \cdots i_{r-1} ; k ; j}^{\alpha}+\sigma_{j i_{1} \cdots i_{r-1} ; k}^{\beta} \Gamma_{\beta j}^{\alpha}\right\} \omega^{i_{1}} \wedge \cdots \wedge \omega^{i_{r-1}}, \\
\left\{D_{k} \mathscr{D}_{j}\left(\sigma \pi \frac{\partial}{\partial \omega^{j}}\right)\right\}^{\alpha}= & \frac{1}{(r-1) !} \sum\left\{\sigma_{j i_{1} \cdots i_{r-1} ; j ; k}^{\alpha}+\Gamma_{\beta j ; k}^{\alpha} \sigma_{j i_{1} \cdots i_{r-1}}^{\beta}\right. \\
& \left.+\Gamma_{\beta j}^{\alpha} \sigma_{j i_{1} \cdots i_{r-1} ; k}^{\beta}-\Lambda_{\gamma k}^{\beta} \Gamma_{\beta j}^{\alpha} \sigma_{j i_{1} \cdots i_{r-1}}^{\gamma}\right\} \omega^{i_{1}} \wedge \cdots \wedge \omega^{i_{r-1}},
\end{aligned}
$$


where $\Lambda=\left(\Lambda_{\alpha k}^{\beta}\right), \Gamma=\left(\Gamma_{\alpha k}^{\beta}\right)$. Then we have the formula

$$
\begin{aligned}
& \sum_{j, k} \omega^{k} \wedge\left\{\mathscr{D}_{j} D_{k}-D_{k} \mathscr{D}_{j}\right\}\left(\sigma \pi \frac{\partial}{\partial \omega^{j}}\right)=\frac{1}{r !} \sum\left\{\left(\Omega_{\beta j i_{s}}^{\alpha}-\delta_{\beta}^{\alpha} R_{i_{s}}^{j}\right)\right. \\
& \left.\cdot \sigma_{i_{1} \cdots i_{s-1} j i_{s+1} \cdots i_{r}}^{\beta}-\frac{1}{2} R_{i_{\mu} i_{\nu} j}^{l} \sigma_{i_{1} \cdots i_{\mu-1} l i_{\mu+1} \cdots i_{\nu-1} j i_{\nu+1} \cdots i_{r}}^{\alpha}\right\} \omega^{i_{1}} \wedge \cdots \wedge \omega^{i_{1}},
\end{aligned}
$$

which proves the statement.

For each $\zeta \in \underline{A_{\mu}^{r}}$ we define the quadratic forms

$$
\tilde{\Omega}(\zeta, \zeta), \hat{\mathscr{R}}(\zeta, \zeta), \mathscr{R}(\zeta, \zeta)
$$

by the formula

$$
\begin{aligned}
\tilde{\Omega}(\zeta, \zeta) & -\hat{\mathscr{R}}(\zeta, \zeta)-\frac{1}{2} \mathscr{R}(\zeta, \zeta) \\
& =\sum_{j, k}\left(\left\{\mathscr{D}_{j} D_{k}-D_{k} \mathscr{D}_{j}\right\} \alpha\left(\zeta \pi \frac{\partial}{\partial \omega^{j}}\right), \alpha\left(\zeta \pi \frac{\partial}{\partial \omega^{k}}\right)\right) .
\end{aligned}
$$

The explicit form of expressions (3.12) is easy to see from the proof of Lemma 3.1.

Now we assume, in what follows, that the manifold $M$ is a finite submanifold of the $C^{\infty}$-manifold $M^{\prime}$. This means that $M$ is an open submanifold of $M^{\prime}$, the closure $\bar{M}$ of $M$ is compact, and the boundary $b M$ of the manifold $M$ is a regularly imbedded $C^{\infty}$ submanifold of $M^{\prime}$ of codimension 1 . Let $M^{\prime}$ be a riemannian manifold, and let $r$ be the $C^{\infty}$ distance function, defined in the neighborhood of $b M$. For $x \in M^{\prime}, r(x)$ is the geodesic distance of $x \in M^{\prime}$ to $b M ; r(x)>0$ if $x \in M, r(x)=0$ if $x \in b M$, and $r(x)<0$ for $x$ in the exterior of $M$.

Let us consider the coordinate neighborhood $U$ on $M^{\prime}$ with the coordinates $\left(x^{1}, \cdots, x^{n}\right)=x, x \in U$, and assume that $U \cap b M \neq \varnothing$. Then

$$
d \boldsymbol{r} \wedge *(d \boldsymbol{r})=d \boldsymbol{r} \cdot d \boldsymbol{r} *(1)=*(1),
$$

where

$$
d \boldsymbol{r}=\frac{\partial \boldsymbol{r}}{\partial \omega^{k}} \omega^{k}
$$

The form $\sigma \in \mathbf{R}_{\mu}^{\mathbf{r}} \mid \mathrm{U}$ is called normal at $x \in b M$ if $d \boldsymbol{r} \wedge \sigma=0$, and the form $\rho \in \mathrm{R}_{\mu}^{\mathrm{r}} \mid \mathrm{U}$ is called tangential at $x \in b M$ if, for each normal $\sigma$ at $x \in b M$, $\left\langle\rho, \overline{\sigma\rangle_{x}}=0\right.$. Thus each $\sigma \in \underline{\mathbf{R}_{\mu}^{r} \mid U}$ can be written in the form

$$
\sigma=t \sigma+n \sigma,
$$

where to is tangential and $n \sigma$ normal at each $x \in b M$. In particular, we get 


$$
t\left(\omega^{1} \wedge \cdots \wedge \hat{\omega}^{l} \wedge \cdots \wedge \omega^{n}\right)=(-1)^{l-1} \frac{\partial \boldsymbol{r}}{\partial \omega^{l}} *(d r)
$$

Let $a, f, g$ be $C^{\infty}$ functions defined on $U$; then

$$
\begin{gathered}
d\left(f a g \omega^{1} \wedge \cdots \wedge \hat{\omega}^{l} \wedge \cdots \wedge \omega^{n}\right) \\
=(-1)^{l-1}\left\{\frac{\partial f}{\partial \omega^{l}} a g+f a \frac{\partial g}{\partial \omega^{l}}+f \frac{\partial a}{\partial \omega^{l}} g\right\} *(1)+f a g(-1)^{l-1} S_{l} *(1),
\end{gathered}
$$

where $S_{l}$ is defined by this formula. Since Stokes' formula can be written in the form

$$
\begin{aligned}
\int_{M} d\left(f a g \omega^{1} \wedge \cdots \wedge \hat{\omega}^{l} \wedge \cdots \wedge \omega^{n}\right) & =\int_{b M} f a g t\left(\omega^{1} \wedge \cdots \wedge \hat{\omega}^{l} \wedge \cdots \wedge \omega^{n}\right) \\
& =(-1)^{l-1} \int_{b M} f a g \frac{\partial r}{\partial \omega^{l}} *(d r)
\end{aligned}
$$

we have from (3.13) the formulas:

$$
\begin{aligned}
\int_{M} f a \frac{\partial g}{\partial \omega^{k}} *(1)= & -\int_{M} \frac{\partial f}{\partial \omega^{k}} a g *(1)+\int_{b M} f a g \frac{\partial \boldsymbol{r}}{\partial \omega^{k}} *(d r) \\
& -\int_{M} f a g S_{k} *(1)-\int_{M} f \frac{\partial a}{\partial \omega^{k}} g *(1), \\
\int_{M} \frac{\partial f}{\partial \omega^{k}} a g *(1)= & -\int_{M} f a \frac{\partial g}{\partial \omega^{k}} *(1)+\int_{b M} f a g \frac{\partial \boldsymbol{r}}{\partial \omega^{k}} *(d \boldsymbol{r}) \\
& -\int_{M} f a g S_{k} *(1)-\int_{M} f \frac{\partial a}{\partial \omega^{k}} g *(1) .
\end{aligned}
$$

Moreover on the basis of the identity

$$
\frac{\partial^{2}}{\partial \omega^{k} \partial \omega^{j}}+c_{k j}^{h} \frac{\partial}{\partial \omega^{h}}=\frac{\partial^{2}}{\partial \omega^{j} \partial \omega^{k}}+c_{j k}^{h} \frac{\partial}{\partial \omega^{h}}
$$

we get

$$
\begin{aligned}
\int_{M} \frac{\partial f}{\partial \omega^{j}} a \frac{\partial g}{\partial \omega^{k}} *(1)= & \int_{M} \frac{\partial f}{\partial \omega^{k}} a \frac{\partial g}{\partial \omega^{j}} *(1) \\
& +\int_{b M} f a\left[\frac{\partial g}{\partial \omega^{k}} \frac{\partial \boldsymbol{r}}{\partial \omega^{j}}-\frac{\partial g}{\partial \omega^{j}} \frac{\partial \boldsymbol{r}}{\partial \omega^{k}}\right] *(d \boldsymbol{r}) \\
& +\int_{M} f a\left(c_{j k}^{h}-c_{k j}^{h}\right) \frac{\partial g}{\partial \omega^{h}} *(1)
\end{aligned}
$$




$$
\begin{aligned}
& +\int_{M} f a\left(\frac{\partial g}{\partial \omega^{j}} S_{k}-\frac{\partial g}{\partial \omega^{k}} S_{j}\right) *(1) \\
& +\int_{M} f\left(\frac{\partial a}{\partial \omega^{k}} \frac{\partial g}{\partial \omega^{j}}-\frac{\partial a}{\partial \omega^{j}} \frac{\partial g}{\partial \omega^{k}}\right) *(1) .
\end{aligned}
$$

\section{The $D$-Neumann problem}

In this section we formulate the $D$-Neumann problem, and briefly indicate the Hilbert spaces associated with it.

Let us now assume that $\mathscr{D}$ is an elliptic differential operator, and let $D^{*}$ be the formal adjoint to the operator $D$ in the resolution (2.7) of the sheaf $\Theta$. This means that, for any $v \in \underline{C}_{\mu}^{\mathrm{r}-1}$ with compact support in $M^{\prime}$, and each $u \in \mathrm{C}_{\mu}^{\mathrm{r}}$ the identity $\left(D^{*} u, v\right)=(\overline{u, D} v)$ holds, where $($,$) is the global product$ on $\bar{M}^{\prime}$. Considering now the representation of $u \in \underline{C_{\mu}^{r}}$ by the pair $(\sigma, \zeta)$, we have the formulas

$$
\begin{aligned}
& D u=D(\sigma, \zeta)=\left(D_{0} \sigma-\zeta, D_{0}\left(D_{0} \sigma-\zeta\right)\right), \\
& D^{*} u=D_{*}(\sigma, \zeta)=\left(D_{0}^{*}\left(\sigma+D_{0}^{*} \zeta\right),-\alpha\left(\sigma+D_{0}^{*} \zeta\right)\right) .
\end{aligned}
$$

The first formula is part of Proposition 2.2, and the second follows by straightforward computation. It is easy to see that $D_{0}^{2}$ and $D_{0}^{* 2}$ are linear maps over the local rings of $C^{\infty}$-functions. Then we have the well-defined mapping

$$
=D D^{*}+D^{*} D: \underline{\mathrm{C}}_{\mu}^{\mathrm{r}} \rightarrow \underline{\mathrm{C}}_{\mu}^{\mathrm{r}},
$$

which is called the laplacian.

Let us assume further that $\mu$ is a fixed integer and that $\mu \geq \mu_{1}$. Proposition 2.4 shows that the laplacian $\square$ is elliptic if and only if the operator $\mathscr{D}$ is elliptic in the stable range.

We introduce the following notation:

C : the restriction of the space of sections of $C_{\mu}=\oplus_{r} C_{\mu}^{r}$ to $\bar{M}$, elements which are smooth up to and including the boundary;

$C_{0} \quad$ : the completion of $C$ in the norm \|\| ;

$D, D^{*}$ : the extensions to $C_{0}$ of the operators $D, D^{*}$ in Spencer's sequence (we keep the same notation despite the fact that the spaces on which these operators are defined are different, and the spaces will be specified if necessary.)

$C_{1} \quad$ : the elements of $C_{0}$ which are in the intersection of the domains of $D$ and $D^{*}$;

$N \quad$ : the elements $u \in C_{0}$ such that $D u$ lies in the domain of $D^{*}$, and $D^{*} u$ lies in the domain of $D$; 
$\boldsymbol{H} \quad$ : the subspace of $N$ composed of the elements of $N$ which are annihilated by the laplacian $L=D D^{*}+D^{*} D(L$ is actually the Friedrichs extension of the laplacian $\square$ on $N$ ).

We observe that these spaces have the structure of graded vector spaces, for example $\boldsymbol{H}=\oplus_{r} \boldsymbol{H}^{r}$.

Definition 3.1. We say that the weak Neumann problem is solvable for a finite manifold $M$, and the elliptic differential operator $\mathscr{D}$, if $L N$ is a closed subspace of $\boldsymbol{C}_{0}$.

We observe that the operator $L$ is self-adjoint and that $(L+I)^{-1}$ is a bounded operator on $C_{0}$. We define next the orthogonal projection

$$
H: C_{0} \rightarrow H
$$

If the weak Neumann problem is solvable, we have the orthogonal decomposition

$$
C_{0}=D D^{*} N \oplus D^{*} D N \oplus H,
$$

i.e., for each $u \in C_{0}, u=L w+H w$, where $w \in N$. We define the map (Neumann operator)

$$
N: C_{0} \rightarrow N
$$

by the relation: $N u=w-H w$.

Proposition 3.1. The weak Neumann problem is solvable if and only if the operators $H$ and $N$ have the following properties: $N$ is a self-adjoint bounded operator satisfying

$$
\begin{gathered}
N H=H N=0, \\
u=D D^{*} N u+D^{*} D N u+H u
\end{gathered}
$$

for each $u \in C_{0}$.

Proof. The proof follows essentially the lines of the proof of Proposition $2.8[8]$.

For each $u, v \in C_{1}$ we define the Dirichlet inner product

$$
Q(u, v)=(D u, D v)+\left(D^{*} u, D^{*} v\right)+(u, v),
$$

and the norm $Q(u)$ by $Q(u)^{2}=Q(u, u)$. If the norm $Q$ is completely continuous with respect to the norm \|\| and this is expected to be true generally only in positive degrees, then the weak Neumann problem is solvable, or solvable in positive degrees. But it seems that the relation $D N=N D$ lies deeper.

Definition 3.2. We say that the $D$-Neumann problem is solvable if the weak Neumann problem is solvable and if the Neumann operator $N$ com- 
mutes with $D$, (i.e., if $u$ belongs to the domain of $D$, then $N u$ also belongs to the domain of $D$ and $N D u=D N u)$.

Proposition 3.2. If, for each $u \in C_{1}$, the irregularity

$$
Q^{\prime}(u, u) \geq \varepsilon \int_{b M}|u|_{x}^{2} *(d \boldsymbol{r})
$$

holds for some $\varepsilon>0$ then the D-Neumann problem is solvable and

$$
H \cong Z\left(C_{0}\right) / D\left(C_{0}\right)
$$

where $Z\left(C_{0}\right)$ is the kernel of the map $D: C_{0} \rightarrow C_{0}$, and $\boldsymbol{H}^{r}$ is a finite dimensional vector space for $r \geq 1$.

Proof. That the solvability of the $D$-Neumann problem follows from the assumption of the proposition is a consequence of the fundamental theorems proved in the paper [9].

If the $D$-Neumann problem is solvable, we have the Neumann decomposition

$$
u=D\left(D^{*} N\right) u+\left(D^{*} N\right) D u+H u
$$

for each $u \in C_{0}$. This decomposition provides a cochain homotopy

$$
1-H=D\left(D^{*} N\right)+\left(D^{*} N\right) D,
$$

and the isomorphism (3.22) follows.

The inequality (3.21), together with the ellipticity, implies the complete continuity in positive degrees, of the Dirichlet norm $Q$ with respect to the norm \|\| . This finite dimensionality of the harmonic spaces in positive degrees follows from a standard argument.

Let $\boldsymbol{H}=\oplus_{r} \boldsymbol{H}^{r}$ be the space of elements $u \in C$, which satisfy the conditions

$$
\begin{aligned}
& \left(D^{*} u, v\right)=(u, D v), \\
& \left(D^{*} D u, v\right)=(D u, D v)
\end{aligned}
$$

for all $v \in C$, and which are annihilated by the laplacian $\square$. The solvability of the $D$-Neumann problem implies the isomorphism $\boldsymbol{H}^{r} \cong \boldsymbol{H}^{r}, r \geq 1$.

Remark. (i) There is another cohomology, namely the cohomology defined by the formula

$$
\mathscr{H}=Z(C) / D(C),
$$

where $C$ is the space of sections of $C_{\mu}=\oplus_{r} C_{\mu}^{r}$ over $M$. The relation between $\mathscr{H}=\oplus_{r} \mathscr{H}^{r}$ and $\boldsymbol{H}=\oplus_{r} \boldsymbol{H}^{r}$ requires further investigation even if the Neumann problem has been solved, although these spaces are probably isomorphic in positive degrees, as was conjectured by D.C. Spencer.

(ii) The condition (3.21) is not necessary for the solvability of the $D$ Neumann problem, and a weaker condition, for example, 


$$
Q(u, u) \geq c\|u\|_{(s)} \text { for } 0<s<\frac{1}{2}, c>0, u \in C_{1}
$$

may suffice (see [14]). But for our purpose (vanishing theorem) these conditions are not very important.

One of the main problems is to determine the set $C\left(\mathscr{D}_{\mu_{0}}\right)$ of finite manifolds for which the $D$-Neumann problem is solvable. The manifolds of $C\left(\mathscr{D}_{\mu_{0}}\right)$ would be in some sense, generalizations to elliptic operators of the classical holomorphy domains (see [12]).

W. J. Sweeney has recently shown that the Neumann problem for an elliptic system with constant coefficients on a spherical domain is not always solvable in terms of the euclidean metric.

\section{Vanishing of the harmonic space in positive degrees for open manifolds}

The harmonic space $H$ is the subspace of $C$ composed of those elements, which satisfy both boundary conditions and are annihilated by the laplacian $\square$. It would then be enough to calculate an explicit form of the Dirichlet integral yielding a fundamental inequality for a vanishing theorem for elements satisfying both boundary conditions. Nevertheless, we give a formula for the Dirichlet integral under somewhat more general conditions, namely, we assume that the elements are smooth up to and including the boundary and satisfy only the first boundary condition (3.24).

In view of the remark made at the end of the preceding section, the significance of this section, so far as vanishing of cohomology is concerned, is dubious.

Let $\left\{U_{\alpha}\right\}$ be a finite covering of $\bar{M}$ by coordinate neighborhoods in $M^{\prime}$, and $U$ be a neighborhood of this covering such that $U \cap b M \neq \varnothing$.

Proposition 3.3. Let $u$ be a section of $C_{\mu}^{r}$ over $U$ which is smooth up to and including the boundary $b U$ of $U$ and is such that the first boundary condition (3.24) is satisfied. Then, for the elements $\sigma, \zeta$ is the representation $(\sigma, \zeta)$ of $u$, and the following identities hold on $b U$ :

$$
\begin{gathered}
\sum_{j=1}^{n} \frac{\partial r}{\partial \omega^{j}}\left(\sigma \frac{\partial}{\partial \omega^{j}}\right)=0, \\
\sum_{j=1}^{n} \frac{\partial r}{\partial \omega^{j}}\left(\zeta \frac{\partial}{\partial \omega^{j}}\right)=0, \\
\frac{\partial^{2} \boldsymbol{r}}{\partial \omega^{k} \partial \omega^{l}}\left(\sigma \pi \frac{\partial}{\partial \omega^{l}}\right)+\frac{\partial}{\partial \omega^{l}} \frac{\partial}{\partial \omega^{k}}\left(\sigma \pi \frac{\partial}{\partial \omega^{l}}\right)=\lambda \frac{\partial \boldsymbol{r}}{\partial \omega^{k}}, \\
\frac{\partial^{2} \boldsymbol{r}}{\partial \omega^{k} \partial \omega^{l}} \alpha\left(\zeta \pi \frac{\partial}{\partial \omega^{l}}\right)+\frac{\partial \boldsymbol{r}}{\partial \omega^{l}} \frac{\partial}{\partial \omega^{k}} \alpha\left(\zeta \pi \frac{\partial}{\partial \omega^{l}}\right)=\tilde{\lambda} \frac{\partial \boldsymbol{r}}{\partial \omega^{k}} .
\end{gathered}
$$


Proof. Let $u \in \underline{C_{\mu}^{r} \mid U}, v \in \underline{C_{\mu}^{r-1} \mid U}$, and let $u$ belong to the domain of the operator $D^{*}$; then, using the representation $u=(\sigma, \zeta), v=(\rho, \eta)$, we have by Proposition 2.2 the formula

$$
\left\langle D^{*} u, v\right\rangle *(1)-\langle u, D v\rangle *(1)=d\{\sigma \wedge a * \rho-\zeta \wedge a * \eta\} .
$$

Since (3.24) holds for such $u \in C$ and all $v \in C$, we have the identity

$$
\int(\sigma \wedge a * \rho-\zeta \wedge a * \eta)=0
$$

Thus

$$
t(\sigma \wedge a * \rho)=t(\zeta \wedge a * \eta)=0
$$

on $b U$, i.e.,

$$
d \boldsymbol{r} \wedge \sigma \wedge a * \rho=0, \quad d \boldsymbol{r} \wedge \zeta \wedge a * \eta=0 .
$$

The last identities are equivalent to

$$
\left.\sigma \pi \frac{\partial}{\partial \boldsymbol{r}}\right|_{\partial U}=0,\left.\alpha\left(\zeta \pi \frac{\partial}{\partial \boldsymbol{r}}\right)\right|_{\partial U}=0 .
$$

Since

$$
\frac{\partial}{\partial \boldsymbol{r}}=\sum_{j} \frac{\partial \boldsymbol{r}}{\partial \omega^{j}} \frac{\partial}{\partial \omega^{j}},
$$

we obtain (3.25), (3.26). The equations (3.27) and (3.28) are obtained by derivation of (3.25) and (3.26).

Definition 3.3. The finite manifold $M$ will be said to be "strongly pseudoconvex" with respect to the differential operator $\mathscr{D}$ if at each point of the boundary $b M$, the condition

$$
\sum_{i, j=1}^{n} L_{i j}\left\{\alpha\left(\zeta \pi \frac{\partial}{\partial \omega^{i}}\right), \alpha\left(\zeta \pi \frac{\partial}{\partial \omega^{j}}\right)\right\rangle>0,
$$

where

$$
L_{i j}=\nabla_{j} \nabla_{i} \boldsymbol{r}
$$

is satisfied for all $\zeta$ satisfying (3.26).

Remark. The condition (3.29) is too restrictive in general to be the right "Levi criterion" for the determination of an existence domain (analogue of holomorphy domain) for an elliptic operator.

Lemma 3.2. For any $u \in C$ which satisfies the first boundary condition (3.24), we have the following identity: 
(3.30) $\|D u\|^{2}+\|D * u\|^{2}+\|u\|^{2}=K(u, u)+T(u, u)+\int_{b M} L_{0}(u, u) *(d r)$

where

$$
\begin{aligned}
& K(u, u)=\Omega(\sigma, \sigma)-\tilde{R}(\sigma, \sigma)-\frac{1}{2} R(\sigma, \sigma) \\
& +\tilde{\Omega}(\zeta, \zeta)-\mathscr{R}(\zeta, \zeta)-\frac{1}{2} \mathscr{R}(\zeta, \zeta) \text {. } \\
& L_{0}(u, u)=\sum_{k, j=1}^{n} \frac{\partial^{2} \boldsymbol{r}}{\partial \omega^{k} \partial \omega^{j}}\left\{\alpha\left(\zeta \pi \frac{\partial}{\partial \omega^{k}}\right), \alpha\left(\zeta \pi \frac{\partial}{\partial \omega^{j}}\right)\right\} \\
& +\sum_{k, j=1}^{n} \frac{\partial^{2} \boldsymbol{r}}{\partial \omega^{k} \partial \omega^{j}}\left\{\sigma \pi \frac{\partial}{\partial \omega^{k}}, \sigma \pi \frac{\partial}{\partial \omega^{j}}\right\}, \\
& T(u, u) \sum_{k}\left\|D_{k} \zeta\right\|^{2}+\sum_{k}\left\|D_{k} \sigma\right\|^{2}-\left(\beta\left(D_{k} \zeta \pi \frac{\partial}{\partial \omega^{j}}\right), \beta\left(D_{j} \zeta \pi \frac{\partial}{\partial \omega_{k}}\right)\right) \\
& -2\left(\alpha D_{k}\left(\zeta \pi \frac{\partial}{\partial \omega^{j}}\right), \alpha \sum C_{j k}^{n}\left(\zeta \pi \frac{\partial}{\partial \omega^{h}}\right)\right) \\
& +\left(\left(\left(\alpha D_{0}^{*}\right) \zeta,\left\{f_{k} \alpha+2 \psi_{k}+\alpha \bar{f}_{k}+2 \alpha \tilde{\Delta}_{k}\right\}\left(\zeta \pi \frac{\partial}{\partial \omega^{k}}\right)\right)\right. \\
& -2\left(D_{0}^{*} \sigma,\left\{\bar{f}_{k}-d_{k}\right\}\left(\sigma \pi \frac{\partial}{\partial \omega^{k}}\right)\right) \\
& -2\left(D_{k}\left(\sigma \pi \frac{\partial}{\partial \omega^{j}}\right), \sum C_{j k}^{\iota}\left(\sigma \pi \frac{\partial}{\partial \omega^{l}}\right)\right) \\
& +\left(\zeta \pi \frac{\partial}{\partial \omega^{k}},\left\{\left(\tilde{f}_{j}+2 \tilde{\Delta}_{j}\right) D_{k} \alpha-D_{k} \alpha\left(\bar{f}_{j}+2 \tilde{\Delta}_{j}\right)\right.\right. \\
& \left.\left.-\Delta_{j} \Delta_{k}+\widetilde{d_{k} \alpha} \phi_{j}-\psi_{j} \phi_{j}+\sum C_{l h}^{k} \alpha C_{h l}^{j}\right\}\left(\zeta \pi \frac{\partial}{\partial \omega^{j}}\right)\right) \\
& +\left(\sigma \pi \frac{\partial}{\partial \omega^{k}},\left\{\bar{f}_{j} \Lambda_{k}-D_{k} \bar{f}_{j}+\sum C_{l h}^{k} C_{h l}^{j}\right\}\left(\sigma \pi \frac{\partial}{\partial \omega^{j}}\right)\right) \\
& -2\left(\zeta, D_{0} \sigma\right)-2\left(D_{0} \zeta, D_{0}^{2} \sigma\right)+2\|\zeta\|^{2}+\left\|D_{0}^{2} \sigma\right\|^{2}+2\left(D_{0}^{*} \sigma, D_{0}^{* 2} \zeta\right) \\
& +2\left(\alpha \sigma, \alpha D_{0}^{* \zeta}\right)+\left\|D_{0}^{* 2} \zeta\right\|^{2}+\|\alpha \sigma\|^{2}+\|\sigma\|^{2} \text {, }
\end{aligned}
$$

where

$$
\begin{gathered}
\Delta_{k}=D_{k} \alpha-\alpha D_{k}, \quad \phi_{k}=\mathscr{D}_{k} \alpha-\alpha \mathscr{D}_{k}, \quad \tilde{\Delta}_{k}=a \Delta_{k} a^{-1}, \quad \tilde{\phi}_{k}=a \phi_{k} a^{-1}, \\
d_{k}=\tilde{\Lambda}_{k}+\Lambda_{k}+\Gamma_{k}-S_{k}-\theta_{k}, \quad f_{k}=-\Lambda_{k}-\tilde{\Lambda}_{k}-\tilde{\Gamma}_{k}+S_{k}+\theta_{k}, \cdots .
\end{gathered}
$$

Proof. For each $u=(\sigma, \zeta) \in C$ satisfying the first boundary condition there are the formulas 


$$
\begin{gathered}
\|D u\|^{2}=\left\|D_{0} \sigma\right\|^{2}+\left\|D_{0} \zeta\right\|^{2}-2\left(\zeta, D_{0} \sigma\right)-2\left(D_{0} \zeta, D_{0}^{2} \sigma\right)+\|\zeta\|^{2}+\left\|D_{0}^{2} \sigma\right\|^{2}, \\
\left\|D^{*} u\right\|^{2}= \\
=D_{0}^{*} \sigma\left\|^{2}+\right\| \alpha D_{0}^{*} \zeta \|^{2}+2\left(D_{0}^{*} \sigma, D_{0}^{* 2} \xi\right) \\
+2\left(\alpha \sigma, \alpha D_{0}^{*} \zeta\right)+\left\|D_{0}^{* 2} \zeta\right\|^{2}+\|\alpha \sigma\|^{2} .
\end{gathered}
$$

By definition of the adjoint $D_{0}^{*}$ of $D_{0}$ we have

$$
\begin{aligned}
& \left\|D_{0}^{*} \sigma\right\|^{2}=\left(\mathscr{D}_{j}\left(\sigma \pi \frac{\partial}{\partial \omega^{j}}\right), \mathscr{D}_{k}\left(\sigma \pi \frac{\partial}{\partial \omega^{k}}\right)\right), \\
& \left\|D_{0} \sigma\right\|^{2}=\sum_{j}\left\|D_{j} \sigma\right\|^{2}-\left(D_{j} \sigma \pi \frac{\partial}{\partial \omega^{k}}, D_{k} \sigma \pi \frac{\partial}{\partial \omega^{j}}\right) .
\end{aligned}
$$

Using the formulas (3.14) for integration by parts, the formulas (3.27), (3.28) and the identity

$$
\left(\frac{\partial}{\partial \omega^{l}} \sigma\right) \pi \frac{\partial}{\partial \omega^{k}}-\frac{\partial}{\partial \omega^{l}}\left(\sigma \pi \frac{\partial}{\partial \omega^{k}}\right)=\sum_{h} c_{l k}^{h}\left(\sigma \pi \frac{\partial}{\partial \omega^{h}}\right),
$$

which is easily proved to be valid for $\sigma \in R_{\mu}^{r}$ and any $r$, we obtain the formulas stated in Lemma 3.2 by straightforward calculation.

Theorem 3.1. If the curvature of the operator $\mathscr{D}$ is such that the quadratic form $K(u, u)$ is sufficiently positive for all $u \in C^{\tau}, r \geq 1$, and if both conditions (3.24) and condition (3.29) are satisfied, then

$$
\boldsymbol{H}^{r}=0, \quad r \geq 1
$$

Proof. Since $H^{r}=H^{r}, r \geq 1$, it is enough to consider elements smooth up to and including the boundary $b M$. If we write $T^{\prime}(u, u)=T(u, u)-\left\|^{\prime}\right\|^{2}$, we obtain from (3.30) for a harmonic element $u \in C$ the identity

$$
K(u, u)+T^{\prime}(u, u)+\int_{b M} L(u, u) * d r=0 .
$$

And from here we can conclude that if $K(u, u)$ is sufficiently positive in a sense that the left side of the above equality is $>0$, then $u=0$.

\section{Vanishing of the cohomology groups $\boldsymbol{H}^{r}, r \geq 1$, for a compact manifold}

We continue to assume that the operator $\mathscr{D}$ is elliptic and assume, in addition, that the manifold is compact. Moreover, we assume that the resolution (2.7) is exact for $\mu \geq \mu_{1}$, i.e., the resolution of the sheaf $\Theta$ of germs of solutions of the homogeneous equation by the fine sheaves $C_{\mu}^{r}, \mu \geq \mu_{1}$, is exact. Let $\mu$ be a fixed integer $\mu \geq \mu_{1}$, and let $C=\oplus_{r} C^{r}$ be the graded vector space of sections of $C_{\mu}=\oplus_{r} C_{\mu}^{r}$ over the manifold. Then 


$$
H^{r}(M, \Theta) \cong Z\left(C^{r}\right) / D\left(C^{r-1}\right)
$$

(de Rham's theorem). Let

$$
\boldsymbol{H}^{r}=\left\{u \mid u \in C^{r}, D u=0, D^{*} u=0\right\}
$$

be the harmonic forms of degree $r$; then

$$
\boldsymbol{H}^{r}(M, \Theta) \cong \boldsymbol{H}^{r}
$$

(Hodge's theorem). In fact, on the compact manifold the Neumann problem is solvable by standard arguments and this yields the isomorphism (3.35). Our purpose here is to give a vanishing theorem for the harmonic spaces in positive degrees, i.e. by (3.35) for the cohomology in positive degrees.

The desired fundamental inequality can be derived from the formula (3.30) for the Dirichlet integral. However, by restricting our attention only to harmonic elements, we can proceed to the inequality somewhat more directly. Let $u=(\sigma, \zeta)$ be an element of $C^{r}$ in obvious identification. If this jet form is harmonic, then $D u=D^{*} u=0$ and, by (3.15), $D_{0} \sigma=\zeta, D_{0}^{*} \sigma=-D_{0}^{* 2} \zeta$ and $\alpha \sigma=-\alpha D_{0}^{*} \zeta$. Thus, for any harmonic $u=(\sigma, \zeta)$, we have

$$
\begin{aligned}
& \left(D_{0} D_{0}^{*}+D_{0}^{*} D_{0}\right) \sigma-D_{0}^{*} \zeta+D_{0} D_{0}^{* 2} \zeta=0, \\
& \left(D_{0} \alpha D_{0}^{*}+\alpha D_{0}^{*} D_{0}\right) \zeta+D_{0} \alpha \sigma-\alpha D_{0}^{*} D_{0}^{2} \sigma=0 .
\end{aligned}
$$

The "laplacian" $D_{0} D_{0}^{*}+D_{0}^{*} D_{0}$ is explicitly given by (3.8), and the following formula can be regarded as the Weitzenböck formula for the "laplacian" $D_{0} \alpha D_{0}^{*}+\alpha D_{0}^{*} D_{0}$ :

$$
\begin{aligned}
& \left(D_{0} \alpha D_{0}^{*}+\alpha D_{0}^{*} D_{0}\right) \zeta=-\alpha \sum_{j} \mathscr{D}_{j} D_{j} \zeta+\alpha\left\{\omega_{k} \wedge \mathscr{D}_{j} D_{k} \beta\left(\zeta \pi \frac{\partial}{\partial \omega^{j}}\right)\right\} \\
& \quad+\omega^{k} \wedge\left\{\mathscr{D}_{j} D_{k}-D_{k} \mathscr{D}_{j}\right\} \alpha\left(\zeta \pi \frac{\partial}{\partial \omega^{j}}\right)-\omega^{k} \wedge D_{k}\left\{\alpha \mathscr{D}_{j}-\mathscr{D}_{j} \alpha\right\}\left(\zeta \pi \frac{\partial}{\partial \omega^{j}}\right) \\
& \quad+\alpha\left\{\left(\sum c_{j i}^{k} \omega^{i}+\Lambda_{j} \omega^{k}+\Gamma_{j} \omega^{k}\right) \wedge\left(D_{k} \zeta \pi \frac{\partial}{\partial \omega^{j}}\right)\right\} \\
& \quad+\alpha\left\{\omega^{k} \wedge \mathscr{D}_{j} \sum c_{j k}^{k}\left(\zeta \pi \frac{\partial}{\partial \omega^{n}}\right)\right\} .
\end{aligned}
$$

Since, for any harmonic $u=(\sigma, \zeta),(3.36)$ holds, we have the identitiss:

$$
\begin{aligned}
& \left(\left(D_{0} D_{0}^{*}+D_{0}^{*} D_{0}\right) \sigma, \sigma\right)-\left(D_{0}^{*} \zeta, \sigma\right)+\left(D_{0} D_{0}^{* 2} \zeta, \sigma\right)=0, \\
& \left(\left(D_{0} \alpha D_{0}^{*}+\alpha D_{0}^{*} D_{0}\right) \zeta, \zeta\right)+\left(D_{0} \alpha \sigma, \zeta\right)-\left(\alpha D_{0}^{*} D_{0}^{2} \sigma, \zeta\right)=0 .
\end{aligned}
$$

Straightforward calculation gives

Lemma 3.3. For any harmonic jet form $u=(\sigma, \zeta) \in C^{r}, r \geq 1$, where $(\sigma, \zeta)$ is the obvious representation of $u$, the following identities hold: 


$$
\begin{aligned}
& \Omega(\sigma, \sigma)-\frac{1}{2} R(\sigma, \sigma)-\hat{R}(\sigma, \sigma) \\
& =-\sum_{j}\left\|D_{j} \sigma\right\|^{2}+\left(D_{0}^{* \zeta}, \sigma\right)-\left(D_{0} D_{0}^{* 2} \zeta, \sigma\right), \\
& \tilde{\Omega}(\zeta, \zeta)-\frac{1}{2} \mathscr{R}(\zeta, \zeta)-\hat{\mathscr{R}}(\zeta, \zeta)=-\sum_{j}\left\|D_{j} \zeta\right\|^{2}+\left\|\beta D_{0}^{*} \zeta\right\|^{2} \\
& +\left(\left\{\mathscr{D}_{j} \beta-\beta \mathscr{D}_{j}\right\}\left(\zeta \pi \frac{\partial}{\partial \omega^{j}}\right), \beta \mathscr{D}_{k}\left(\zeta \pi \frac{\partial}{\partial \omega^{k}}\right)\right) \\
& +\left(\mathscr{D}_{j} \beta\left(\zeta \pi \frac{\partial}{\partial \omega^{j}}\right),\left\{\mathscr{D}_{k} \beta-\beta \mathscr{D}_{k}\right\}\left(\zeta \pi \frac{\partial}{\partial \omega^{k}}\right)\right) \\
& -\int_{M}\left\{\left\{\frac{\partial}{\partial \omega^{j}} \beta\left(\zeta \pi \frac{\partial}{\partial \omega^{j}}\right), \beta\left(\zeta \pi \frac{\partial}{\partial \omega^{k}}\right)\right)\right. \\
& \left.-\left\{\frac{\partial}{\partial \omega^{j}} \beta\left(\zeta \pi \frac{\partial}{\partial \omega^{k}}\right), \beta\left(\zeta \pi \frac{\partial}{\partial \omega^{j}}\right)\right\}\right\} S_{k} *(1) \\
& \text { (3.37) } \quad-\left(\frac{\partial}{\partial \omega^{j}} \beta\left(\zeta \pi \frac{\partial}{\partial \omega^{j}}\right), \theta_{k} \beta\left(\zeta \pi \frac{\partial}{\partial \omega^{k}}\right)\right) \\
& +\left(\frac{\partial}{\partial \omega^{j}} \beta\left(\zeta \pi \frac{\partial}{\partial \omega^{k}}\right), \theta_{k} \beta\left(\zeta \pi \frac{\partial}{\partial \omega^{j}}\right)\right) \\
& +\left(\left\{c_{j k}^{h}-c_{k j}^{h}\right\} \frac{\partial}{\partial \omega^{h}} \beta\left(\zeta \pi \frac{\partial}{\partial \omega^{k}}\right), \beta\left(\zeta \pi \frac{\partial}{\partial \omega^{j}}\right)\right) \\
& +2\left(\Lambda_{k} \beta\left(\zeta \pi \frac{\partial}{\partial \omega^{j}}\right), D_{j} \beta\left(\zeta \pi \frac{\partial}{\partial \omega^{k}}\right)\right) \\
& -2\left(\left\{\Lambda_{k}+\Gamma_{k}\right\} \beta\left(\zeta \pi \frac{\partial}{\partial \omega^{k}}\right), \mathscr{D}_{j} \beta\left(\zeta \pi \frac{\partial}{\partial \omega^{j}}\right)\right) \\
& +\left(\omega^{k} \wedge D_{k}\left\{\alpha \mathscr{D}_{j}-\mathscr{D}_{j} \alpha\right\}\left(\zeta \pi \frac{\partial}{\partial \omega^{j}}\right), \zeta\right) \\
& -\left(\left\{\sum c_{j i}^{k} \omega^{i}+\Lambda_{j} \omega^{k}+\Gamma_{j} \omega^{k}\right\} \wedge\left\{D_{k} \zeta \pi \frac{\partial}{\partial \omega^{j}}\right\}, \zeta\right) \\
& -\left(\omega^{k} \wedge \mathscr{D}_{j} \sum c_{k j}^{h}\left(\zeta \pi \frac{\partial}{\partial \omega^{h}}\right), \zeta\right)-\left(D_{0} \alpha \sigma, \zeta\right)+\left(\alpha D_{0}^{*} D_{0}^{2} \sigma, \zeta\right) .
\end{aligned}
$$

Lemma 3.4. If $\mathscr{D}$ is an elliptic operator, then for any harmonic jet form $u=(\sigma, \zeta) \in C^{r}, r \geq 1$, there exist positive constants $K_{1}, K_{2}$ such that

$$
K(u, u) \leq K_{1}\|\sigma\|^{2}+K_{2}\|\zeta\|^{2},
$$

where

$$
\begin{aligned}
K(u, u)= & \Omega(\sigma, \sigma)-\frac{1}{2} R(\sigma, \sigma)-\hat{R}(\sigma, \sigma) \\
& +\tilde{\Omega}(\zeta, \zeta)-\frac{1}{2} \mathscr{R}(\zeta, \zeta)-\mathscr{R}(\zeta, \zeta)
\end{aligned}
$$


Proof. It is enough to prove this inequality locally, for $u$ with compact support in some fixed neighborhood $U$ of a finite covering $\left\{U_{\alpha}\right\}$ of the compact manifold $M$.

We shall consider first the term $\sum_{j}\left\|D_{j} \zeta\right\|^{2}-\left\|\beta D_{0}^{*} \zeta\right\|^{2}$. Reasoning similar to that in Proposition 2.6 shows that the ellipticity of a differential operator $\mathscr{D}$ is equivalent to the existence of a constant $c, o<c<1$, such that

$$
\left\|\left(\beta d^{*}\right) \zeta\right\|^{2} \leq(1-c) \sum_{j}\left\|d_{j} \zeta\right\|^{2}
$$

for any $\zeta \in \underline{A_{\mu}^{r}}, \mu \geq \mu_{1}, r \geq 1$, with compact support in $U, \sum d x^{j} \wedge d_{j} \zeta=d \zeta$ Then, from the identity

$$
\begin{gathered}
\left\|\beta D_{0}^{* \zeta}\right\|^{2}-\sum_{j}\left\|D_{j} \zeta\right\|^{2}=-\sum_{j}\left\|d_{j} \zeta\right\|^{2}+\left(\beta d_{k}\left(\zeta \pi \frac{\partial}{\partial x^{k}}\right), \beta d_{j}\left(\zeta \pi \frac{\partial}{\partial x^{j}}\right)\right) \\
-2 \sum_{j}\left(D_{j} \zeta, \Lambda_{j} \zeta\right)+2\left(\beta\left\{\Lambda_{j}+\Gamma_{j}\right\}\left(\zeta \pi \frac{\partial}{\partial x^{j}}\right), \beta \mathscr{D}_{k}\left(\zeta \pi \frac{\partial}{\partial x_{k}}\right)\right)
\end{gathered}
$$

we obtain the inequality

$$
\begin{aligned}
& \left\|\beta D_{0}^{*} \zeta\right\|^{2}-\sum_{j}\left\|D_{j} \zeta\right\|^{2} \leq-c \sum_{j}\left\|d_{j} \zeta\right\|^{2}-2 \sum_{j}\left(D_{j} \zeta, \Lambda_{j} \zeta\right) \\
& \quad+2\left(\beta\left\{\Lambda_{j}+\Gamma_{j}\right\}\left(\zeta \pi \frac{\partial}{\partial x^{j}}\right), \beta_{k}\left(\zeta \pi \frac{\partial}{\partial x^{k}}\right)\right) .
\end{aligned}
$$

Let us observe now the following elementary fact. For any positive number $\varepsilon$ there exists a positive constant $K(\varepsilon)$ such that, for any two elements $\sigma, \rho \in R_{\mu}^{r}$, we have $(\sigma, \rho) \leq \varepsilon\|\sigma\|^{2}+K(\varepsilon)\|\rho\|^{2}$. We then derive from the formulas (3.37), for some $\varepsilon_{1}>0, \varepsilon_{2}>0, \varepsilon_{3}>0$, the following inequalities :

$$
\begin{aligned}
& \Omega(\sigma, \sigma)-\frac{1}{2} R(\sigma, \sigma)-\hat{R}(\sigma, \sigma) \leq-\sum_{j}\left\|D_{j} \sigma\right\|^{2}+\varepsilon_{1} \sum_{j}\left\|D_{j} \zeta\right\|^{2}+K\left(\varepsilon_{1}\right)\|\sigma\|^{2}, \\
& \tilde{\Omega}(\zeta, \zeta)-\frac{1}{2} \mathscr{R}(\zeta, \zeta)-\hat{\mathscr{R}}(\zeta, \zeta) \leq\left\|\beta D_{0}^{*} \zeta\right\|^{2}-\sum_{j}\left\|D_{j} \zeta\right\|^{2}+\varepsilon_{2} \sum_{j}\left\|D_{j} \zeta\right\|^{2} \\
& +\varepsilon_{3} \sum\left\|D_{j} \sigma\right\|^{2}+K\left(\varepsilon_{2}, \varepsilon_{3}\right)\|\zeta\|^{2} \text {. }
\end{aligned}
$$

Adding these two inequalities, and using (3.40) we obtain (3.38).

From the inequality (3.38) we infer the following:

Theorem 3.2. Let $M$ be a compact manifold and $\mathscr{D}$ an elliptic differential operator. If the quadratic form $K(u, u)$ is sufficiently positive for all $u \in C^{r}$, $r \geq 1$, then

$$
\boldsymbol{H}^{r}(M, \Theta)=0, \quad r \geq 1 .
$$

Remark. The condition that "the quadratic form $K(u, u)$ is sufficiently 
positive" is equivalent to the statement "the curvature of the operator $\mathscr{D}$ is sufficiently positive with respect to the curvature of the riemannian metric on $M$ "'.

We were using so far only the resolution (2.7) of the sheaf $\Theta$. Considering the resolution (2.10), we are led to the laplacian $D_{0}^{*} \beta D_{0}+\beta D_{0} D_{0}^{*}$, the corresponding Weitzenböck formula and another formulation of the vanishing theorem for a compact manifold and elliptic differential operators. The condition for vanishing of the cohomology groups (3.42) is then given by sufficient positivity of the quadratic form $K(u, u)$ on sections of the bundles $B_{\mu}^{r}, r \geq 1$, i.e. on elements $u=(\beta \sigma, 0)$ in the natural isomorphism and representation of $u$ by a pair as in Proposition 2.2.

Under the assumptions we have made at the beginning of this section, we can conclude from Theorem 2.1 that the resolution (2.10) is exact. Then the cohomology $H(M, \Theta)$ is isomorphic to the space $B=\oplus_{r} B^{r}$ of sections over $M$ of $B_{\mu}=\oplus_{r} B_{\mu}^{r}$, where $\mu$ is fixed, $\mu \geq \mu_{1}$, which are annihilated by the laplacian $D_{0}^{*} \beta D_{0}+B D_{0} D_{0}^{*}$. Let $\rho \in B$; then the Weitzenböck formula has the form

$$
\begin{aligned}
\left(D_{0}^{*} \beta D_{0}+\right. & \left.\beta D_{0} D_{0}^{*}\right) \rho \\
= & -\mathscr{D}_{j} \beta D_{j} \rho+\beta\left\{\omega^{k} \wedge\left\{\mathscr{D}_{j} D_{k}-D_{k} \mathscr{D}_{j}\right\} \beta\left(\rho \pi \frac{\partial}{\partial \omega^{j}}\right)\right\} \\
& +\beta\left\{\omega^{k} \wedge\left\{\mathscr{D}_{j} D_{k}-D_{k} \mathscr{D}_{j}\right\} \alpha\left(\rho \pi \frac{\partial}{\partial \omega^{j}}\right)\right\} \\
& +\omega^{k} \wedge\left\{\mathscr{D}_{j} \beta-\beta \mathscr{D}_{j}\right\} D_{k}\left(\rho \pi \frac{\partial}{\partial \omega^{j}}\right) \\
& +\left\{\sum c_{j e}^{k} \omega^{e}+\Lambda_{j} \omega^{k}+\Gamma_{j} \omega^{k}\right\} \wedge \beta D_{k}\left(\rho \pi \frac{\partial}{\partial \omega^{j}}\right) \\
& -\mathscr{D}_{j}\left\{\left[\beta\left(\omega^{k} \wedge D_{k} \rho\right)-\left(\omega^{k} \wedge \beta D_{k} \rho\right)\right] \pi \frac{\partial}{\partial \omega^{j}}\right\} \\
& +\mathscr{D}_{j}\left\{\omega^{k} \wedge \beta \sum c_{k j}^{h}\left(\rho \pi \frac{\partial}{\partial \omega^{j}}\right)\right\} \\
& +\mathscr{D}_{j}\left\{\omega^{k} \wedge\left[\beta\left(D_{k} \rho \pi \frac{\partial}{\partial \omega^{j}}\right)+\left(\beta D_{k} \rho \pi \frac{\partial}{\partial \omega^{j}}\right)\right]\right\} .
\end{aligned}
$$

Using this formula, we have the following

Lemma 3.5. For any $\rho \in B^{r}, r \geq 1$ the identity

$$
\begin{gathered}
\Omega_{\beta}(\rho, \rho)- \\
-\frac{1}{2} R_{\beta}(\rho, \rho)-\hat{R}_{\beta}(\rho, \rho)=\left(\left\{D_{0}^{*} \beta D_{0}+\beta D_{0} D_{0}^{*}\right\} \rho, \rho\right) \\
+\left\|\alpha D_{0} \rho\right\|^{2}-\sum_{j}\left\|D_{j} \rho\right\|^{2}+\left(\beta D_{j} \rho, \Lambda_{j} \rho\right)
\end{gathered}
$$




$$
\begin{aligned}
& -\left(\beta D_{j} \rho \theta_{j} \rho\right)-\int_{M}\left\langle\beta D_{j} \rho, \rho\right\rangle S_{j} *(1) \\
& +\left(\alpha \sum c_{k j}^{e}\left(\rho \pi \frac{\partial}{\partial \omega^{e}}\right), \alpha D_{j}\left(\rho \pi \frac{\partial}{\partial \omega^{k}}\right)\right) \\
& +\left(\alpha D_{k} \rho \pi \frac{\partial}{\partial \omega^{j}}, \alpha \sum c_{j k}^{e}\left(\rho \pi \frac{\partial}{\partial \omega^{e}}\right)\right) \\
& -\left(\left\{D_{k} \alpha-\alpha D_{k}\right\}\left(\rho \pi \frac{\partial}{\partial \omega^{j}}\right), D_{j}\left(\rho \pi \frac{\partial}{\partial \omega^{k}}\right)\right) \\
& +\left(\left\{\mathscr{D}_{j} \alpha-\alpha \mathscr{D}_{j}\right\}\left(\rho \pi \frac{\partial}{\partial \omega^{j}}\right), \mathscr{D}_{j}\left(\rho \pi \frac{\partial}{\partial \omega^{k}}\right)\right) \\
& -\left(\left\{D_{k} \beta-\beta D_{k}\right\}\left(\rho \pi \frac{\partial}{\partial \omega^{j}}\right), D_{j} \alpha\left(\rho \pi \frac{\partial}{\partial \omega^{k}}\right)\right) \\
& -\left(\beta D_{k}\left(\rho \pi \frac{\partial}{\partial \omega^{j}}\right),\left\{D_{j} \alpha-\alpha D_{j}\right\}\left(\rho \pi \frac{\partial}{\partial \omega^{k}}\right)\right) \\
& +\left(\left\{\mathscr{D}_{j} \beta-\beta \mathscr{D}_{j}\right\}\left(\rho \pi \frac{\partial}{\partial \omega^{j}}\right), \mathscr{D}_{k} \alpha\left(\rho \pi \frac{\partial}{\partial \omega^{k}}\right)\right) \\
& +\left(\beta \mathscr{D}_{j}\left(\rho \pi \frac{\partial}{\partial \omega^{j}}\right),\left\{\mathscr{D}_{k} \alpha-\alpha \mathscr{D}_{k}\right\}\left(\rho \pi \frac{\partial}{\partial \omega^{k}}\right)\right) \\
& -\left(\left\{\mathscr{D}_{j} \beta-\beta \mathscr{D}_{j}\right\} D_{k}\left(\rho \pi \frac{\partial}{\partial \omega^{j}}\right), \rho \pi \frac{\partial}{\partial \omega^{k}}\right) \\
& -\left(\mathscr{D}_{j}\left\{\omega^{k} \wedge \beta \sum c_{k j}^{h}\left(\rho \pi \frac{\partial}{\partial \omega^{h}}\right)\right\}, \rho\right) \\
& -\left(\left\{\sum c_{j e}^{k} \omega^{e}+\Lambda_{j} \omega^{k}+\Gamma_{j} \omega^{k}\right\} \wedge \beta D_{k}\left(\rho \pi \frac{\partial}{\partial \omega^{j}}\right), \rho\right) \\
& -\left(\mathscr{D}_{j}\left\{\omega^{k} \wedge\left[\beta\left(D_{k} \rho \pi \frac{\partial}{\partial \omega^{j}}\right)-\left(\beta D_{k} \rho \pi \frac{\partial}{\partial \omega^{j}}\right)\right]\right\}, \rho\right) \\
& -\left(\mathscr{D}_{j}\left\{\left[\left(\omega^{k} \wedge \beta D_{k} \rho\right)-\beta\left(\omega^{k} \wedge D_{k} \rho\right)\right] \pi \frac{\partial}{\partial \omega^{j}}\right\}, \rho\right) \text {, }
\end{aligned}
$$

holds, where

$$
\begin{aligned}
& \Omega_{\beta}(\rho, \rho)-\frac{1}{2} R_{\beta}(\rho, \rho)-\hat{R}_{\beta}(\rho, \rho) \\
& \quad=\left(\left\{\mathscr{D}_{j} D_{k}-D_{k} \mathscr{D}_{j}\right\} \beta\left(\rho \pi \frac{\partial}{\partial \omega^{j}}\right), \beta\left(\rho \pi \frac{\partial}{\partial \omega^{k}}\right)\right) .
\end{aligned}
$$

We shall write

$$
K_{\beta}(\rho, \rho)=\Omega_{\beta}(\rho, \rho)-\frac{1}{2} R_{\beta}(\rho, \rho)-\hat{R}_{\beta}(\rho, \rho) .
$$


Theorem 3.3. Let $M$ be a compact manifold and $\mathscr{D}$ an elliptic operator. If the quadratic form $\Omega_{\beta}(\rho, \rho)$ is sufficiently positive in the sense that $K_{\beta}(\rho, \rho)$ is sufficiently positive, then

$$
H^{r}(M, \Theta)=0, \quad r \geq 1
$$

Proof. The statement follows immediately from the inequality

$$
\Omega_{\beta}(\rho, \rho)-\frac{1}{2} R_{\beta}(\rho, \rho)-\hat{R}_{\beta}(\rho, \rho) \leq+k\|\rho\|^{2},
$$

which holds for all $\rho \in B^{r}, r \geq 1$, for some positive constant $k$. From (3.44) follows the inequality for $\varepsilon>0, K(\varepsilon)>0$,

$$
\begin{aligned}
& \Omega_{\beta}(\rho, \rho)-\frac{1}{2} R_{\beta}(\rho, \rho)-\hat{R}_{\beta}(\rho, \rho) \leq\left\|\alpha D_{0} \rho\right\|^{2} \\
& \quad-\sum_{j}\left\|D_{j} \rho\right\|^{2}+\varepsilon \sum_{j} a_{j}\left\|D_{j} \rho\right\|^{2}+K(\varepsilon)\|\rho\|^{2},
\end{aligned}
$$

where $a_{j}$ in general depends on the metric chosen along the fibres of $R_{\mu}$ and on the splitting of the exact sequence (2.9). Since $\mathscr{D}$ is an elliptic operator, we have the inequality

$$
\left\|\alpha D_{0} \rho\right\|^{2}-\sum_{j}\left\|D_{j} \rho\right\|^{2} \leq C \sum_{j}\left\|D_{j} o\right\|^{2}+K_{1}\|\rho\|^{2},
$$

which holds for all $\rho \in B^{r}, C$ and $K_{1}$ being positive constants. This proves the inequality (3.45) and hence the theorem.

\section{An example}

Let $M$ be a compact manifold of complex dimension $m(n=2 m)$, and $T_{c}(M)$ the complex tangent bundle of $M$. We have the obvious isomorphism $T_{c}(M) \cong V \oplus \bar{V}$, where $V$ is the bundle of holomorphic tangent vectors on $M$. Further let $E$ be a complex line bundle, and let

$$
\mathscr{D}: \underline{E} \rightarrow \bar{V}^{*},
$$

where $\bar{V}^{*}$ is the dual bundle of $\bar{V}$, the first order differential operator which has in local complex coordinates $z=\left(z^{1}, \cdots, z^{n}\right)$ on $U \subset M$ the form

$$
\mathscr{D} s=\sum_{\nu} \frac{\partial s}{\partial \bar{z}^{\nu}} d \bar{z}^{\nu}
$$

The vector bundle $R_{1}$, as sub-bundle of $J_{1}(E)$, is locally given as the set of elements of the form $\left(s, \frac{\partial s}{d z^{\nu}}, \frac{\partial s}{\partial \bar{z}^{\nu}}\right)$ for which $\frac{\partial s}{\partial \bar{z}^{\nu}}=0$ holds. The operator $\mathscr{D}$ is an involutive and elliptic operator. In this case $\mu_{0}=\mu_{1}=1$ and every element $u=(\sigma, \xi) \in \underline{\mathrm{C}_{0}^{\mathrm{r}}}=\underline{\mathrm{R}_{1}^{\mathrm{r}} / \mathrm{A}_{1}^{\mathrm{r}}}$ in the representation of Proposition 2.1 is a 
pair of independent elements, where $\sigma \in \underline{\mathrm{R}_{0}^{\mathrm{r}}} \approx \mathrm{E} \oplus \wedge^{\mathrm{p}} \Lambda^{*} \wedge^{\mathrm{q}} \overline{\mathrm{V}}^{*}, p+q=r$, and $\xi$ belongs to the ideal of $\mathrm{E} \oplus \wedge^{p^{\prime}} \mathrm{V}^{*} \wedge^{q^{\prime}} \overline{\mathrm{V}}^{*}, p^{\prime}+q^{\prime}=r+1$ generated by $\mathrm{E} \oplus \mathrm{V}^{*}$, in the sense of exterior algebra. The differential operator $D$ (see Proposition 2.1) splits into two operators, namely $D=D_{1}+D_{2}$, where

$$
D_{1}(\sigma, \xi)=(\partial \sigma-\xi,-\partial \xi), \quad D_{2}(\sigma, \xi)=(\bar{\partial} \sigma,-\bar{\partial} \xi)
$$

and we have the exact commutative diagram (see [12])

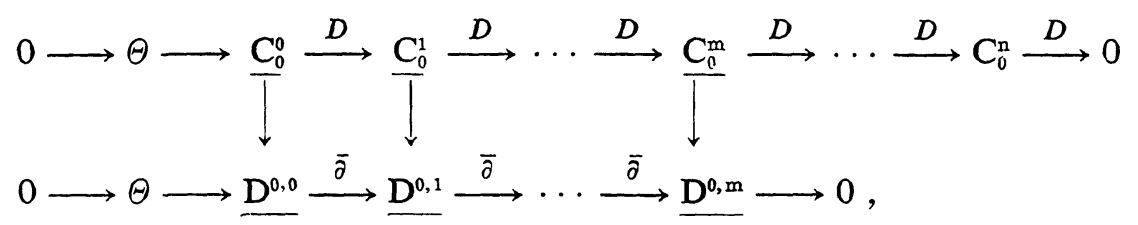

where $\Theta$ is the sheaf of germs of holomorphic functions on $M$, and the second sequence is the classical Dolbeault resolution of $\Theta$ by sheaves of complexvalued differential forms.

The splitting $\lambda$ of the exact sequence (2.9) can be chosen, in this case in such a way that $D_{0}$ is the covariant differential of some metric connection corresponding to a Hermitian metric in $E$. Then $D_{\Gamma}=D_{0}$ (see (3.4)), because $\Gamma=0$, and the curvature $\Omega$ of the differential operator $\mathscr{D}$ is just the curvature of the corresponding Hermitian metric. Theorem 1 of [7]-Kodaira's vanishing theorem-then follows from Theorem 3.3 of this paper.

\section{THE BOTT CONNECTION}

The construction of the bundles $C_{\mu}^{r}, \mu \geq \mu_{1}, r=0,1, \cdots, n$ of jet-forms, given in Proposition 2.3, is due essentially to $\mathrm{R}$. Bott. The construction was originally formulated in terms of a so called Bott connection or, in the paper [2], on the basis of a connection from $R_{\mu}$ to $R_{\mu-1}$. Such a connection is given by a "derivation" $D$ in Definition 2.1 . In this section, we shall give some characterizations of these connections, using the principal bundles associated to the considered vector bundles. Because the operator $D$ is well defined for all $\mu \geq 1$ we shall not make any restrictions on $\mu$.

Let $q: P_{p} \rightarrow M$ be the principal bundle associated to the vector bundle $p: R_{\mu} \rightarrow M$, and let $G_{\mu}$ be the structure group. Let $\mathcal{S}_{\mu}$ be the Lie algebra of $G_{\mu}$. Denoting by $V_{\mu}$ the typical fibre of $R_{\mu}$, we have the commutative diagram

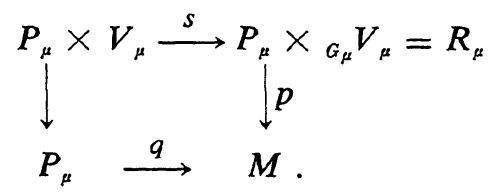


Assume that there are given the following mappings : projection $\pi: R_{\mu} \rightarrow R_{\mu-1}$, homomorphisms $h: G_{\mu} \rightarrow G_{\mu-1}$ and $k: V_{\mu} \rightarrow V_{\mu-1}$ such that $k(g v)=h(g) k(v)$ for all $g \in G_{\mu}$ and any $v \in V_{\mu}$. There exists the bundle map $l: P_{\mu} \rightarrow P_{\mu-1}$ compatible with the operation of the group on the principal bundle. Namely, for $(z, v) \in P_{\mu} \times V_{\mu}, \pi \circ s(z, v) \in R_{\mu-1}$, there exists a unique $z^{\prime} \in P_{\mu-1}$ such that $s^{\prime}\left(z^{\prime}, k(v)\right)=\pi \circ s(z, v)$, where $s^{\prime}$ is defined by the diagram

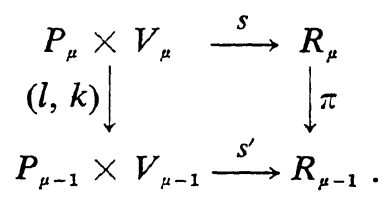

We then define $z^{\prime}=l(z)$.

For any $X \in T(M)$, we denote by $\bar{X} \in T\left(P_{\mu}\right)$ a vector such that $q_{*} \bar{X}=X$. The vector field $\bar{X} \in \mathrm{T}\left(\mathrm{P}_{\mu}\right)$ which projects on $X \in \mathrm{T}(\mathrm{M})$ is called a projectable vector field. Let $U \overline{\subset M}$ be any neighborhood over which $R_{\mu}$ is trivial. If $\sigma \in \Gamma\left(U, R_{\mu}\right)$, then there is a unique section $\sigma^{\prime} \in \Gamma\left(q^{-1}(U), P_{\mu} \times V_{\mu}\right)$, such that $s \circ \sigma^{\prime}=\sigma \circ q$. The section $\sigma^{\prime}$ is given by a $V_{\mu}$-valued function $\tilde{\sigma}$ on $P_{\mu}$, in the sence that for any $z \in q^{-1}(U), \sigma^{\prime}(z)=(z, \tilde{\sigma}(z))$ holds. It is clear that $\tilde{\sigma}(z g)=g^{-1} \tilde{\sigma}(z)$ for any $g \in G_{\mu}$. We shall speak about $V_{\mu}$-valued $G_{\mu}$-functions on $P_{\mu}$. There is a one-one correspondence between the sections of $R_{\mu}$ and the sections of the sheaf of germs of $V_{\mu}$-valued $G_{\mu}$-functions over $M$.

Definition 4.1. A Bott connection from $R_{\mu}$ to $R_{\mu-1}$ is given by the first order operator

$$
D_{\mu}: \underline{\mathbf{R}_{\mu}^{\mathrm{r}}} \rightarrow \underline{\mathbf{R}_{\mu-1}^{\mathrm{r}+1}}, \quad r=0,1, \cdots, n-1,
$$

where the conditions (i) and (ii) of Definition 2.1 are satisfied.

Remark. This definition is actually exactly Definition 2.1. The only diference is that we use the symbol $D_{\mu}$ instead of $D$ in order to make clear that the Bott connection on the $\mu$-th level is considered.

The "lifting" of the section $\sigma \in \underline{\mathbf{R}_{\mu}}$ into the $G_{\mu}$-functions on $P_{\mu}$ will always be denoted by $\tilde{\sigma}$. A long tilde over several symbols will be omitted for typographical reasons, but its presence should be clear from the context.

Proposition 4.1. To the Bott connection $D_{\mu}$ and any projectable vector field $\bar{X}$ on $P_{\mu}$, there is associated an element $\omega(\bar{X}) \in \operatorname{Hom}\left(V_{\mu}, V_{\mu-1}\right)$ by the formula

$$
\omega(\bar{X}) \tilde{\sigma}=i\left(q_{*} \bar{X}\right) D_{\mu} \sigma-\sigma \in \underline{\mathbf{R}_{\mu}} .
$$

The element $\omega(\bar{X})$, considered as a mapping of $V_{\mu}$-valued $G_{\mu}$-functions on $P_{\mu}$, is linear over functions and satisfies the following two conditions:

(i) $\omega(\tilde{\lambda})=k \lambda$, where $\tilde{\lambda}$ is a fundamental vector field on $P_{\mu}$ corresponding 
to $\lambda \in \mathrm{CS}_{\mu}$, and the composition on the right side is the composition of endomorphism $\lambda$ on $V_{\mu}$ and projection $k$.

$$
\omega\left(\bar{X}_{z g}\right)=h(g)^{-1} \omega\left(\bar{X}_{z}\right) g .
$$

And any Bott connection can be given by such a 1-form $\omega$ which satisfies (i) and (ii).

Proof. Straight from the definition it follows that $\omega(\bar{X})$ is an element of $\operatorname{Hom}\left(V_{\mu}, V_{\mu-1}\right)$.

Let $\bar{f}$ be a lifting from $M$ onto $P_{\mu}$ of a real-valued function $f$. Then

$$
\begin{aligned}
\omega(\bar{X}) \bar{f} \tilde{\sigma} & =i(X) D_{\mu}(f \sigma)-k \bar{X}(\bar{f} \tilde{\sigma}) \\
& =\bar{f} i(X) D_{\mu} \sigma+i(X) d f \otimes \pi \sigma-d \bar{f}(\bar{X}) \otimes k \tilde{\sigma}-\bar{f} k \bar{X} \tilde{\sigma} \\
& =\bar{f} \omega(\bar{X}) \tilde{\sigma},
\end{aligned}
$$

so $\omega(\bar{X})$ is linear over functions.

The fundamental vector field $\tilde{\lambda}$ on $P_{\mu}$ corresponds to some element of the Lie algebra $\mathbb{S}_{\mu}$ of $G_{\mu}$ in the homomorphism of the Lie algebra $\mathbb{B}_{\mu}$ into the vector fields on $P_{\mu}$, given by the action of $G_{\mu}$ on $P_{\mu}$. Then if $\gamma$ is a 1 -form on $P_{\mu}$ with values in $\mathbb{S}_{\mu}$, such that the restriction to the fibre is the Cartan form, we have the identity $\gamma(\tilde{\lambda})=\lambda$. And since for any fundamental vector field $\tilde{\lambda}$ on $P_{p}$ the formula (4.2) has the form

$$
\omega(\tilde{\lambda}) \tilde{\sigma}(z)=-k \tilde{\lambda} \tilde{\sigma}(z), \quad z \in P_{\mu},
$$

we obtain the relation

$$
\begin{aligned}
\omega(\tilde{\lambda}) \tilde{\sigma}\left(z_{0} g\right) & =-k \tilde{\lambda} \tilde{\sigma}\left(z_{0} g\right)=-k \tilde{\lambda}\left(g^{-1} \tilde{\sigma}\left(z_{0}\right)\right)=-k \tilde{\lambda}\left(g^{-1}\right) \tilde{\sigma}\left(z_{0}\right) \\
& =-k\left(d g^{-1}(\tilde{\lambda})\right) \tilde{\sigma}\left(z_{0}\right)=k\left(\left(g^{-1} d g\right) \tilde{\lambda}\right) g^{-1} \tilde{\sigma}\left(z_{0}\right)=k \lambda \tilde{\sigma}\left(z_{0}\right),
\end{aligned}
$$

where $z_{0}$ is a fixed point of $P_{\mu}$. This proves the identity (i).

The formula (4.2) and the diagram (4.1) give the identities

$$
\begin{aligned}
s(l(z g), & \left.\omega\left(\bar{X}_{z g}\right) \tilde{\sigma}(z g)\right)=s\left(l(z g), i\left(q_{*} \bar{X}\right) D_{\mu} \sigma(z g)\right)-s(l(z g), k \bar{X} \tilde{\sigma}(z g)) \\
= & s\left(l(z) h(g), i\left(q_{*} \bar{X}\right) h(g)^{-1} D_{\mu} \sigma(z)\right)-s\left(l(z) h(g), h^{-1}(g) k \bar{X} \tilde{\sigma}(z)\right) \\
= & s\left(l(z), i\left(q_{*} \bar{X}\right) D_{\mu} \sigma(z)\right)-s(l(z), k \bar{X} \tilde{\sigma}(z))=s\left(l(z), \omega\left(\bar{X}_{z}\right) \tilde{\sigma}(z)\right) .
\end{aligned}
$$

In this formula the subscript $z$ is added to $\bar{X}$ for better understanding. It is immediate that (ii) follows from the relation

$$
s\left(l(z g), \omega\left(\bar{X}_{z g}\right) \tilde{\sigma}(z g)\right)=s\left(l(z), h(g) \omega\left(\bar{X}_{z g}\right) g^{-1} \tilde{\sigma}(z)\right) .
$$

And now we shall prove the reverse statement. Observe that the Bott connection on the $\mu$-th level gives the linear mapping $v\left(\bar{X}_{z}\right)$, associated to the vector $\bar{X}_{z} \in T_{z}\left(P_{\mu}\right)$, of a local section $\sigma$ of $R_{\mu}$ over some neighborhood of $q(z)$ into the fibre of $R_{\mu}$ over $q(z)$. This mapping is defined by the formula 


$$
v\left(\bar{X}_{z}\right) \sigma=s\left(l(z), \omega\left(\bar{X}_{z}\right) \tilde{\sigma}\right) .
$$

we then have the relation

$$
\begin{aligned}
v\left(\bar{X}_{z}\right) \sigma & =s\left(l(z), i\left(q_{*} \bar{X}\right) D_{\mu} \sigma-k \bar{X} \tilde{\sigma}\right) \\
& =s\left(l(z), i\left(q_{*} \bar{X}\right) D_{\mu} \sigma\right)-s(l(z), k \bar{X} \tilde{\sigma}) \\
& =i\left(q_{*} \bar{X}\right) D_{\mu} \sigma-\pi s(z, \bar{X} \tilde{\sigma})
\end{aligned}
$$

From these formulas it follows that the operator $D_{\mu}$ gives a Bott connection.

Let $W_{\mu}$ be the subspace of $\operatorname{Hom}\left(V_{\mu}, V_{\mu-1}\right)$ generated by elements of the form $\omega(\tilde{\lambda})$, where $\tilde{\lambda}$ is some projectable vector field on $P_{\mu}$. If we denote also by $\mathbb{S}_{\mu}$ the representation of the Lie algebra $\mathcal{S S}_{\mu}$ in $V_{\mu}$, which is induced by the action of $G_{\mu}$ in $V_{\mu}$, we have :

Proposition 4.2. $W_{\mu}=k \circ$ SS $_{\mu}$.

Proof. To the splitting $\tau$ of the exact sequence

$$
0 \rightarrow g_{\mu+1} \rightarrow R_{\mu+1} \rightarrow R_{\mu} \rightarrow 0
$$

and the Bott connection $D_{\mu}$, there is associated the connection on the vector bundle $R_{\mu}$ defined by the operator $D_{\mu+1} \circ \tau$. We have namely the relation

$$
D_{\mu}=D_{\mu} \circ \pi \circ \tau=\pi \circ D_{\mu+1} \circ \tau \text {. }
$$

It is easily seen that $D_{\mu+1} \circ \tau$ is the covariant differential of an "ordinary" connection on $R_{\mu}$. If $\sigma \in \underline{\mathrm{R}_{\mu}}$, then

$$
\left(D_{\mu+1} \circ \tau\right) f \sigma=D_{\mu+1}(f \tau \sigma)=d f \otimes \sigma+f\left(D_{\mu+1} \circ \tau\right) \sigma .
$$

But the linear mapping $v\left(\bar{X}_{z}\right)$ considered in the proof of Proposition 4.1 satisfies the following:

$$
\begin{aligned}
v\left(\bar{X}_{z}\right) \sigma & =i\left(q_{*} \bar{X}_{z}\right) D_{\mu} \sigma-s\left(l(z), k \bar{X}_{z} \tilde{\sigma}\right) \\
& =i\left(q_{*} \bar{X}_{z}\right) \pi D_{\mu+1} \circ \tau \sigma-s\left(l(z), k \bar{X}_{z} \tilde{\sigma}\right) \\
& =\pi s\left(z, i\left(q_{*} \bar{X}_{z}\right) D_{\mu+1} \circ \tau \sigma\right)-\pi s\left(z, \bar{X}_{z} \tilde{\sigma}\right) \\
& =s\left(l(z), k \circ i\left(q_{*} \bar{X}_{z}\right) D_{\mu+1} \circ \tau \sigma\right)-s\left(l(z), k \circ \bar{X}_{z} \tilde{\sigma}\right)
\end{aligned}
$$

Hence, by (4.3),

$$
\omega(\bar{X}) \tilde{\sigma}=k \circ\left(i\left(q_{*} \bar{X}_{z}\right) D_{\mu+1} \circ \tau \sigma-\bar{X}_{z} \tilde{\sigma}\right) .
$$

Analogously as it was done for the Bott connection, we can find on $P_{\mu}$ the (S) $_{\mu}$-valued 1 -form $\Lambda$ such that

$$
\Lambda(\bar{X}) \tilde{\sigma}=i\left(q_{*} \bar{X}\right) D_{\mu+1} \circ \tau \sigma-\bar{X} \tilde{\sigma} .
$$

It is obvious that $\Lambda$ is a connection form. This concludes the proof. 
Now we shall give another characterization of the Bott connection from $R_{\mu}$ to $R_{\mu-1}$ on the basis of Proposition 4.1. Let us define a mapping

$$
\mathfrak{S}: T\left(G_{\mu}\right) \rightarrow G_{\mu} \times W_{\mu},
$$

by $\mathfrak{S}\left(\lambda_{a}\right)=(a, k \circ \lambda \circ a)$, where $\lambda_{a} \in T_{a}\left(G_{\mu}\right), \lambda$ is the left invariant vector field on $G_{\mu}$ generated by $\lambda_{a}$, and $k \circ \lambda \circ a$ is the composition of homomorphisms. Any section of the trivial bundle $G_{\mu} \times W_{\mu} \rightarrow G_{\mu}$, which is the $\mathfrak{L}$-map of a left invariant vector field on $G_{\mu}$ is said to be left invariant. The space of these sections is isomorphic to $W_{\mu}$ itself. In this case we identify $\mathfrak{S}$ and $k$. Let $A\left(W_{\mu}\right)=\oplus_{i} A^{i}\left(W_{\mu}\right)$ be the exterior algebra of $W_{\mu}^{*}$. The exterior algebra of $W_{\mu}^{*}$. The exterior algebra of differential forms on $P_{\mu}$ is denoted by $E_{\mu}=\oplus_{i} E_{\mu}^{i}$, the element of $W_{\mu}$ corresponding, in the obvious isomorphism, to $\lambda \in \mathbb{S}_{\mu}$ is denoted also by $\lambda$. And let $\tilde{\lambda}$ be the fundamental vector field corresponding to $\lambda$.

Proposition 4.3. The Bott connection on $P_{\mu}$ is a homomorphism (with respect to the exterior multiplication) $\omega^{*}: A\left(W_{\mu}\right) \rightarrow E_{\mu}$, such that

$$
\begin{aligned}
& i\left(\bar{\lambda} \omega^{*}(\chi)=\omega^{*}(i(\lambda) \chi),\right. \\
& \theta(\bar{\lambda}) \omega^{*}(\chi)=\omega^{*}(\theta(\lambda) \chi),
\end{aligned}
$$

for any $\chi \in A\left(W_{\mu}\right)$ and any $\lambda \in \mathbb{S}_{\mu}$. For the interior product and the Lie derivative we use the symbols $i$ and $\theta$.

Proof. We know that the Bott connection on $P_{\mu}$ is given by a $W_{\mu}$-valued 1 -form $\omega$ on $P_{\mu}$-Proposition 4.1. But we know also that $\omega=k \circ \Lambda$ anis there is a homomorphism $\Lambda^{*}: A_{\mu} \rightarrow E_{\mu}\left(A_{\mu} \text { being an exterior algebra ot }\right\}_{*}^{*}$ on $G_{\mu}$ ). Then $\omega^{*}=\Lambda^{*} \circ k^{*}$, where $k^{*}: A\left(W_{\mu}\right) \rightarrow A_{\mu}$ is an obvious homomorphism. We are to prove that, for any $\lambda \in \mathbb{S}_{\mu}$,

$$
i(\tilde{\lambda})\left(\Lambda^{*} \circ k^{*}\right)(\omega)=\left(\Lambda^{*} \circ k^{*}\right)(i(\lambda) \omega), \quad \omega \in A_{\mu} .
$$

But this is equivalent to the identity

$$
i(\tilde{\lambda}) \Lambda^{*}\left(k^{*} \omega\right)=\Lambda^{*}\left(i(\lambda)\left(k^{*} \omega\right)\right),
$$

since $i(\lambda)$ commutes with $k^{*}$. This follows from the fact that $k^{*} \omega$ is the 1 -form of an "ordinary" connection on $P_{\mu}$ (see [1]). Analogously

$$
\theta(\tilde{\lambda}) \Lambda^{*}\left(k^{*} \omega\right)=\Lambda^{*}\left(\theta(\lambda) k^{*} \omega\right),
$$

as we deal with an "ordinary" connection again.

Definition 4.2. The mapping $\Re: A^{\prime}\left(W_{\mu}\right) \rightarrow E_{\mu}^{2}$, where $\Re: \chi \rightarrow d\left(\omega^{*}(\chi)\right)-$ $\omega^{*}(d \chi)$, is called the curvature of the Bott connection $\omega$.

Denote by $S\left(W_{\mu}\right)$ the symmetric algebra associated to $W_{\mu}^{*}$ and by $W_{\mu}=$ $A\left(W_{\mu}\right) \otimes S\left(W_{\mu}\right)$ the so called Weil algebra of $W_{\mu}$. As is easily seen it is 
actually the definition from [1] modified to suit our case. And one would expect to get, on the basis of a chosen Bott connection, a homomorphism $\bar{\Omega}$ of the graded algebra $W_{\mu}$ into $E_{\mu}$ and then the characteristic classes of $P_{\mu}$. But the Bott connections are not useful in this direction, as the following theorem shows.

Theorem 4.1. The curvature of a Bott connection is zero.

Proof. From Proposition 2.2, it follows that $D_{0}^{2}$ is a bundle map from $R_{\mu}^{r}$ into $A_{\mu}^{r+2}$. In the present contex, this means that the curvature form $d \Lambda+[\Lambda, \Lambda]$, where $\omega=k \circ \Lambda$, is a 2-form with values in the kernel of the projection $\mathscr{S}_{\mu} \rightarrow \mathbb{S}_{\mu-1}$. This implies that

$$
k \circ(d \Lambda+[\Lambda, \Lambda])=0 .
$$

Considering the natural extension of $d$ on $W_{\mu}$, we observe that $k$ commutes with the operation $d$. Then, for any $\chi \in A^{\prime}\left(W_{\mu}\right)$,

$$
d\left(\omega^{*}(\chi)\right)-\omega^{*}(d \chi)=d\left(\Lambda^{*} \circ k^{*}(\chi)\right)-\Lambda^{*} d\left(k^{*} \chi\right) .
$$

Using the usual notation $\langle\chi, v\rangle=\chi(v)$, where $\chi \in A\left(W_{\mu}\right)$ and $v \in W_{\mu}$, we can write the following relation

$$
\left\langle\omega^{*}(\chi), \zeta\right\rangle=\langle\chi, \omega(\zeta)\rangle, \quad \chi \in A^{1}\left(W_{\mu}\right), \quad \zeta \in T\left(P_{\mu}\right),
$$

and analogously,

$$
\left\langle d \omega^{*}(\chi), \zeta_{1} \wedge \zeta_{2}\right\rangle=\left\langle\chi, d \omega\left(\zeta_{1}, \zeta_{2}\right)\right\rangle
$$

where

$$
\chi \in A^{1}\left(W_{\mu}\right) ; \zeta_{1}, \zeta_{2} \in T\left(P_{\mu}\right) .
$$

Then, for any $\chi \in A^{\prime}\left(W_{\mu}\right)$ and $\zeta_{1}, \zeta_{2} \in T\left(P_{\mu}\right)$, we have

$$
\begin{aligned}
& \left\langle d\left(\omega^{*}(\chi)\right)-\omega^{*}(d \chi), \zeta_{1} \wedge \zeta_{2}\right\rangle \\
& \left.\quad=\left\langle d\left(\Lambda^{*} \circ k^{*}(\chi)\right)-\left(\Lambda^{*} \circ k^{*}\right) d \chi\right), \zeta_{1} \wedge \zeta_{2}\right\rangle \\
& \quad=\left\langle d\left(\Lambda^{*}\left(k^{*} \chi\right)\right)-\Lambda^{*} d\left(k^{*} \chi\right), \zeta_{1} \wedge \zeta_{2}\right\rangle \\
& \quad=\left\langle k^{*} \chi, d \Lambda\left(\zeta_{1}, \zeta_{2}\right)\right\rangle-\left\langle d\left(k^{*} \chi\right), \Lambda\left(\zeta_{1}\right) \wedge \Lambda\left(\zeta_{2}\right)\right\rangle \\
& =\left\langle k^{*} \chi, d \Lambda\left(\zeta_{1}, \zeta_{2}\right)\right\rangle-\left\langle k^{*} \chi,\left[\Lambda\left(\zeta_{1}\right), \Lambda\left(\zeta_{2}\right)\right]\right\rangle \\
& =\left\langle k^{*} \chi,(d \Lambda+[\Lambda, \Lambda])\left(\zeta_{1}, \zeta_{2}\right)\right\rangle \\
& =\left\langle\chi, k \circ(d \Lambda+[\Lambda, \Lambda])\left(\zeta_{1}, \zeta_{2}\right)\right\rangle=0 .
\end{aligned}
$$

From this formula we conclude that

$$
d\left(\omega^{*}(\chi)\right)-\omega^{*} d(\chi)=0 \text { for any } \chi \in A^{\prime}\left(W_{\mu}\right),
$$

and this yields the statement. 


\section{Bibliography}

[1] H. Cartan, Cohomologie réelle d'un espace fibré principal differentiable, E. N.S. Séminaire de Topologie Algebrique, Paris, 1949-50.

[2] R. Bott, Notes on the Spencer resolution, Mimeographed notes, Harvard University, 1963.

[3] L. Ehrenpreis, V.W. Guillemin \& S. Sternberg, On Spencer's estimate for $\delta$-Poincaré, Ann. of Math. 82 (1965) 128-138.

[4] P.A. Griffiths, Hermitian differential geometry and theory of positive and ample holomorphic vector bundles, J. of Math. Mech. 14 (1965) 117-140.

[5] F. Hirzebruch, Neue topologische Methoden in der algebraischen Geometrie, Springer, Berlin, 1965.

[6] L. Hörmander, Linear partial differential operators, Springer, Berlin, 1963.

[7] K. Kodaira, On a differential-geometric method in the theory of analytic stacks, Proc. Nat. Acad. Sci. U.S.A. 39 (1953) 1268-1273.

[8] J.J. Kohn, Harmonic integrals on strongly pseudoconvex manifolds, I, Ann. of Math. 78 (1963) 112-148.

[9] J. J. Kohn \& L. Nirenberg, Non-coercive boundary value problems, Comm. Pure Appl. Math. 18 (1965) 443-492.

[10] D. G. Quillen, Formal properties of over-determined systems of linear partial differential equations, Harvard thesis, 1964, to appear.

[11] I. M. Singer \& S. Sternberg, The infinite groups of Lie and Cartan, Part I, (The transitive groups), J. Analyse Math. 15 (1965) 1-114.

[12] D. C. Spencer, Deformation of structures on manifolds defined by transitive, continuous pseudogroups III, Ann. of Math. 81 (1965) 389-450.

[13] S. Sternberg, Differential equations, Lectures, Harvard, 1964-65.

[14] W.J. Sweeney, The D-Neumann problem, Stanford thesis, 1966, to appear in Acta Math.

STANFORD UNIVERSITY 